\title{
BANACH SPACES WITH THE 2-SUMMING PROPERTY
}

\author{
A. ARIAS, T. FIGIEL, W. B. JOHNSON AND G. SCHECHTMAN
}

\begin{abstract}
A Banach space $X$ has the 2-summing property if the norm of every linear operator from $X$ to a Hilbert space is equal to the 2-summing norm of the operator. Up to a point, the theory of spaces which have this property is independent of the scalar field: the property is self-dual and any space with the property is a finite dimensional space of maximal distance to the Hilbert space of the same dimension. In the case of real scalars only the real line and real $\ell_{\infty}^{2}$ have the 2-summing property. In the complex case there are more examples; e.g., all subspaces of complex $\ell_{\infty}^{3}$ and their duals.
\end{abstract}

\section{INTRODUCTION}

Some important classical Banach spaces; in particular, $C(K)$ spaces, $L_{1}$ spaces, the disk algebra; as well as some other spaces (such as quotients of $L_{1}$ spaces by reflexive subspaces $[\mathrm{K}],[\mathrm{Pi}]$ ), have the property that every (bounded, linear) operator from the space into a Hilbert space is 2-summing. (Later we review equivalent formulations of the definition of 2-summing operator. Here we mention only that an operator $T: X \rightarrow \ell_{2}$ is 2-summing provided that for all operators $u: \ell_{2} \rightarrow X$ the composition $T u$ is a Hilbert-Schmidt operator; moreover, the 2-summing norm $\pi_{2}(T)$ of $T$ is the supremum of the HilbertSchmidt norm of $T u$ as $u$ ranges over all norm one operators $u: \ell_{2} \rightarrow X$.) In this paper we investigate the isometric version of this property: say that a Banach space $X$ has the 2-summing property provided that $\pi_{2}(T)=\|T\|$ for all operators $T: X \rightarrow \ell_{2}$.

While the 2-summing property is a purely Banach space concept and our investigation lies purely in the realm of Banach space theory, part of the motivation for studying the 2-summing property comes from operator spaces. In [Pa], Paulsen defines for a Banach space $X$ the parameter $\alpha(X)$ to be the supremum of the completely bounded norm of $T$ as $T$ ranges over all norm one operators from $X$ into the space $B\left(\ell_{2}\right)$ of all bounded linear operators on $\ell_{2}$ and asks which spaces $X$ have the property that $\alpha(X)=1$. Paulsen's problem and study of $\alpha(X)$ is motivated by old results of von Neumann, Sz.Nagy, Arveson, and Parrott as well as more recent research of Misra and Sastry. The connection between Paulsen's problem and the present paper is Blecher's

Received by the editors January 26, 1994.

1991 Mathematics Subject Classification. Primary 46B07; Secondary 47A67, 52A10, 52A15.

The first author was supported as a Young Investigator, NSF DMS 89-21369 and NSF DMS 93-21369; the second and fourth authors were workshop participants, NSF DMS 89-21369; the third author was supported by NSF DMS 90-03550 and 93-06376; the third and fourth authors were supported by the U.S.-Israel Binational Science Foundation. 
result [B] that $\alpha(X)=1$ implies that $X$ has the 2-summing property. Another connection is through the property $(\mathrm{P})$ introduced by Bagchi and Misra [BM], which Pisier noticed is equivalent to the 2-summing property. However, since we shall not investigate here $\alpha(X)$ or property $(\mathrm{P})$ directly and do not require results from operator theory, we refer the interested reader to $[\mathrm{Pa}]$ and $[\mathrm{BM}]$ for definitions, history, and references. On the other hand, since the topic we treat here is relevant for operator theorists, we repeat standard background in Banach space theory used herein for their benefit.

In [Pa] Paulsen asks whether $\alpha(X)=1$ only if $X$ is a 1- or 2-dimensional $C(K)$ or $L_{1}$ space; in other words, ignoring the trivial 1-dimensional case, whether $\alpha(X)=1$ implies that $X$ is 2-dimensional, and among 2-dimensional spaces, whether only $\ell_{\infty}^{2}$ and $\ell_{1}^{2}$ satisfy this identity. He proves that $\alpha(X)=1$ implies that $\operatorname{dim}(X)$ is at most 4 , that $\alpha(X)=\alpha\left(X^{*}\right)$, and he gives another proof of Haagerup's theorem that $\alpha\left(\ell_{\infty}^{2}\right)=1$. Paulsen, interested in operator theory, is referring to complex Banach spaces, so $\ell_{\infty}^{2}$ is not the same space as $\ell_{1}^{2}$.

From the point of view of Banach space theory, it is natural to ask which Banach spaces have the 2-summing property both in the real and the complex cases, and here we investigate both questions. Up to a point, the theory is independent of the scalar field: In section 2 we show that the 2 -summing property is self-dual, that only spaces of sufficiently small (finite) dimension can have the property, and that a space with the property is a maximal distance spacethat is, it has maximal Banach-Mazur distance to the Hilbert space of the same dimension. The main result in section 2, Proposition 2.6, gives a useful condition for checking whether a space has the 2-summing property which takes a particularly simple form when the space is 2-dimensional (Corollary 2.7.a).

The analysis in section 3 yields that the situation is very simple in the case of real scalars; namely, $\mathbb{R}$ and $\ell_{\infty}^{2}$ are the only spaces which have the 2 -summing property. Two ingredients for proving this are Proposition 3.1 , which says that there are many norm one operators from real $\ell_{1}^{3}$ into $\ell_{2}^{2}$ which have 2 -summing norm larger than one, and a geometrical argument, which together with a recent lemma of Maurey implies that a maximal distance real space of dimension at least three has a 2-dimensional quotient whose unit ball is a regular hexagon.

The complex case is a priori more complicated, since $\ell_{\infty}^{2}$ and $\ell_{1}^{2}$ both have the 2-summing property but are not isometrically isomorphic. In fact, in section 4 we show that there are many other examples of complex spaces which have the 2-summing property; in particular, $\ell_{\infty}^{3}$ and all of its subspaces. The simplest way to prove that these spaces have the 2-summing property is to apply Proposition 2.6, but we also give direct proofs for $\ell_{\infty}^{3}$ in section 4 and for its 2-dimensional subspaces in the appendix. The case of $\ell_{\infty}^{3}$ itself reduces via a simple but slightly strange "abstract nonsense" argument to a calculus lemma, which, while easy, does not look familiar. (In [BM] the authors give an argument that $\ell_{\infty}^{3}$ satisfies their property (P) which uses a variation of the calculus lemma but replaces the "abstract nonsense" with a reduction to self-adjoint matrices.) We also give in Proposition 4.5 an inequality which is equivalent to the assertion that all 2-dimensional subspaces of complex $\ell_{\infty}^{3}$ have the 2-summing property. While we do not see a simple direct proof of this inequality, we give a very simple proof of a weaker inequality which is equivalent to the assertion 
that every 2-dimensional subspace of complex $\ell_{\infty}^{3}$ is of maximal distance.

In section 5 we make some additional observations.

\section{Preliminaries}

Standard Banach space theory language and results can be found in [LT1], [LT2], while basic results in the local theory of Banach spaces are contained in [T-J2]. However, we recall here that part of the theory and language which we think may not be well known to specialists in operator theory.

Spaces are always Banach spaces, and subspaces are assumed to be closed. Operators are always bounded and linear. The [Banach-Mazur] distance between spaces $X$ and $Y$ is the isomorphism constant, defined as the infimum of $\|T\|\left\|T^{-1}\right\|$ as $T$ runs over all invertible operators from $X$ onto $Y$. The closed unit ball of $X$ is denoted by Ball $(X)$. "Local theory" is loosely defined as the study of properties of infinite-dimensional spaces which depend only on their finite-dimensional spaces, as well as the study of numerical parameters associated with finite-dimensional spaces. Basic for our study and most other investigations in local theory is the fact (see [T-J2, p. 54]) that the distance from an $n$-dimensional space to $\ell_{2}^{n}$ is at most $\sqrt{n}$. One proves this by using the following consequence of F. John's theorem ([T-J2, p. 123]): If $X$ is $n$ dimensional and $\mathscr{E}$ is the ellipsoid of minimal volume containing Ball $(X)$,

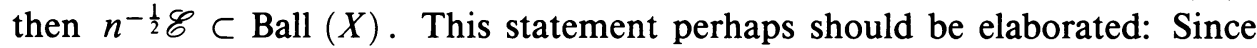
$\operatorname{dim}(X)<\infty$, we can regard $X$ as $\mathbb{R}^{n}$ or $\mathbb{C}^{n}$ with some norm. Among all norm-increasing operators $u$ from $\ell_{2}^{n}$ into $X$, there is by compactness one which minimizes the volume of $u\left(\right.$ Ball $\left.\left(\ell_{2}^{n}\right)\right)$; the distance assertion says that $\left\|u^{-1}\right\| \leq \sqrt{n}$. Alternatively, if one chooses from among all norm one operators from $X$ into $\ell_{2}^{n}$ one which maximizes the volume of the image of Ball $\left(X^{\prime}\right)$, then the norm of the inverse of this operator is at most $\sqrt{n}$. If complex $\ell_{2}^{n}$ is considered as a real space, then it is isometrically isomorphic to real $\ell_{2}^{2 n}$. Thus the distance statement for complex spaces says that a complex space of dimension $n$, when considered as a real space of dimension $2 n$, has (real) distance to (real) $\ell_{2}^{2 n}$ at most $\sqrt{n}$.

Actually, we need more than just the distance consequence of John's theorem. The theorem itself [T-J2, p. 122] says that if $\mathscr{E}$ is the ellipsoid of minimal volume containing Ball $(X)$, then there exist points of contact $y_{1}, \ldots, y_{m}$ between the unit sphere of $X$ and the boundary of $\mathscr{E}$, and there exist positive real numbers $\mu_{1}, \ldots, \mu_{m}$ summing to $\operatorname{dim} X$ so that for each $x$ in $X$, $x=\sum_{i=1}^{m} \mu_{i}\left\langle x, y_{i}\right\rangle y_{i}$, where " $\langle\cdot, \cdot\rangle$ " is the scalar product which generates the ellipsoid $\mathscr{E}$. The existence of many contact points between Ball $(X)$ and $\mathscr{E}$ is important for the proof of Theorem 3.3.

The dual concept to minimal volume ellipsoid is maximal volume ellipsoid. More precisely, an $n$-dimensional space can be regarded as $\mathbb{R}^{n}$ or $\mathbb{C}^{n}$ under some norm $\|\cdot\|$ in such a way that $\operatorname{Ball}(X) \subset \mathscr{E}$, where $\mathscr{E}$ is the usual Euclidean ball and is also the ellipsoid of minimal volume containing Ball $(X)$. Then $X^{*}$ is naturally represented as $\mathbb{R}^{n}$ or $\mathbb{C}^{n}$ under some norm, and the action of $X^{*}$ on $X$ is given by the usual inner product. Then $\mathscr{E}$ is the ellipsoid of maximal volume contained in Ball $\left(X^{*}\right)$.

John's theorem gives many points of contact between Ball $(X)$ and the boundary of the ellipsoid of minimal volume containing Ball $(X)$, and many 
points of contact between the boundary of Ball $(X)$ and the ellipsoid of maximal volume contained in Ball $(X)$. It is a nuisance that these two ellipsoids are not generally homothetic (two ellipsoids are homothetic if one of them is a multiple of the other); however, the situation is better when $X$ has the 2-summing property

Lemma 1.1. Assume that the real or complex $n$-dimensional space $X$ has the 2-summing property and let $\mathscr{E}_{1}$ be the ellipsoid of minimal volume containing Ball $(X)$. Then $n^{-\frac{1}{2} \mathscr{E}_{1}}$ is the ellipsoid of maximal volume contained in Ball $(X)$.

Proof. Let $\mathscr{E}_{2}$ be the ellipsoid of maximal volume contained in Ball $(X)$ and for $i=1,2$ let $|\cdot|_{i}$ be the Euclidean norm which has for its unit ball $\mathscr{E}_{i}$. Let $u_{1}$ be the formal identity map from $X$ to the Euclidean space $\left(X,|\cdot|_{1}\right), u_{2}$ the identity map from $\left(X,|\cdot|_{2}\right)$ to $X$, and let $\lambda_{1}, \ldots, \lambda_{n}$ be the $s$-numbers of the Hilbert space operator $u_{1} u_{2}$ (i.e., the square roots of the eigenvalues of $\left.\left(u_{1} u_{2}\right)^{*} u_{1} u_{2}\right)$. Since $\pi_{2}\left(u_{1}\right)=\left\|u_{1}\right\|=1$ and $\left\|u_{2}\right\|=1$ we have that $\pi_{2}\left(u_{1} u_{2}\right) \leq$ 1. This implies that

$$
\left|\lambda_{1}\right|^{2}+\cdots+\left|\lambda_{n}\right|^{2} \leq 1
$$

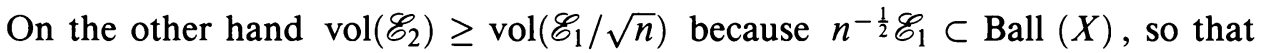
in the case of real scalars we get,

$$
\lambda_{1} \lambda_{2} \cdots \lambda_{n} \geq\left(\frac{1}{\sqrt{n}}\right)^{n},
$$

and in the complex case

$$
\lambda_{1}^{2} \lambda_{2}^{2} \cdots \lambda_{n}^{2} \geq\left(\frac{1}{\sqrt{n}}\right)^{2 n} .
$$

The only way that (1.1) and (1.2) are true is if $\lambda_{1}=\lambda_{2}=\cdots=\lambda_{n}=1 / \sqrt{n}$. But this implies that $\mathscr{E}_{2}=\mathscr{E}_{1} / \sqrt{n}$.

Remark 1.2. B. Maurey has proved a far reaching generalization of Lemma 1.1; namely, that if a space $X$ does not have a unique (up to homothety) distance ellipsoid, then there is a subspace which has the same distance to a Hilbert space as the whole space and which has a unique distance ellipsoid. This implies an unpublished result due to Tomczak-Jaegermann which is stronger than Lemma 1.1 ; namely, that when the distance is maximal, the minimal and maximal volume ellipsoids must be homothetic.

Basic facts about 2-summing operators, and, more generally, $p$-summing operators, can be found in [LT1] and [T-J2]. The 2-summing norm $\pi_{2}(T)$ of an operator from a space $X$ to a space $Y$ is defined to be the supremum of $\left(\sum_{1}^{n}\left\|T U e_{i}\right\|^{2}\right)^{1 / 2}$ where the sup is over all norm one operators $U$ from $\ell_{2}^{n}$, $n=1,2, \ldots$, into $X$ and $\left\{e_{i}\right\}_{i=1}^{n}$ is the unit vector basis for $\ell_{2}^{n}$. When $Y$ is a Hilbert space, this reduces to the definition given in the first paragraph of the introduction, and when $X$ is also a Hilbert space, $\pi_{2}(T)$ is the Hilbert-Schmidt norm of $T$. Note that if $U$ is an operator from $\ell_{2}^{n}$ to a subspace $X$ of $\ell_{\infty}$, then $\|U\|^{2}=\left\|\sum_{i=1}^{n}\left|U e_{i}\right|^{2}\right\|$ [the absolute value is interpreted coordinatewise in $\ell_{\infty}$ ]. So if $T$ goes from $X$ into a space $Y, \pi_{2}(T)$ can be defined intrinsically 
by

$$
\pi_{2}(T)^{2}=\sup \left\{\sum_{i=1}^{n}\left\|T x_{i}\right\|^{2}:\left\|\sum_{i=1}^{n}\left|x_{i}\right|^{2}\right\| \leq 1 ; x_{i} \in X ; n=1,2,3, \ldots\right\} ;
$$

but when $Y$ is an $N$-dimensional Hilbert space, the "sup" is already achieved for $n=N$. (Not relevant for this paper but worth noting is that when $Y$ is a general $N$-dimensional space, the "sup" is achieved for $n \leq N^{2}$ [FLM], [T$\mathrm{J} 2$, p. 141] and is estimated up to the multiplicative constant $\sqrt{2}$ for $n=N$ [T-J1], [T-J2, p. 143].)

It is easy to see that $\pi_{2}$ is a complete norm on the space of all 2-summing operators from $X$ to $Y$ and that $\pi_{2}$ has the ideal property; that is, for any defined composition $T_{1} T_{2} T_{3}$ of operators, $\pi_{2}\left(T_{1} T_{2} T_{3}\right) \leq\left\|T_{1}\right\| \pi_{2}\left(T_{2}\right)\left\|T_{3}\right\|$. The typical 2-summing operator is the formal identity mapping $I_{\infty, 2}$ from $L_{\infty}(\Omega, \mu)$ to $L_{2}(\Omega, \mu)$ when $\mu$ is a finite measure. In this case one gets easily that $\pi_{2}\left(I_{\infty, 2}\right)=\mu(\Omega)^{\frac{1}{2}}$. That such operators are typical is a consequence of the Pietsch factorization theorem ([LT, p. 64], [T-J2, p. 47]), which says that if the space $X$ is isometrically included in a $C(K)$ space, and $T: X \rightarrow Y$ is 2summing, then there is a probability measure $\mu$ on $K$ and an operator $S$ from $L_{2}(K, \mu)$ into $Y$ so that $T$ is the restriction of $S I_{\infty, 2}$ to $X$ and $\|S\|=\pi_{2}(T)$. That is, there is a probability measure $\nu$ on $K$ so that for each $x$ in $X$,

$$
\|T x\|^{2} \leq \pi_{2}(T)^{2} \int\left|x^{*}(x)\right|^{2} d \nu\left(x^{*}\right) .
$$

Of course, the converse to the Pietsch factorization theorem follows from the ideal property for 2-summing operators.

The qualitative version of Dvoretzky's theorem [T-J2, p. 26] says that every infinite dimensional space $X$ contains for every $n$ and $\epsilon>0$ a subspace whose distance to $\ell_{2}^{n}$ is less than $1+\epsilon$. In fact, for a fixed $n$ and $\epsilon$, the same conclusion is true if $\operatorname{dim}(X) \geq N(n, \epsilon)$, and the known estimates for $N(n, \epsilon)$ are rather good.

\section{GenERAL RESUlts}

Here we mention some simple results about spaces which have the 2-summing property, present some motivating examples and then find a characterization of spaces with that property. Let us say that $X$ satisfies the $k$-dimensional 2summing property if $\pi_{2}(T)=\|T\|$ for every operator $T$ from $X$ into $\ell_{2}^{k}$. Thus every space has the 1-dimensional 2-summing property, and $X$ has the 2-summing property if $X$ has the $k$-dimensional 2-summing property for every positive integer $k$. We introduce this definition because our techniques suggest that a space with the 2-dimensional 2-summing property has the 2-summing property, but we cannot prove this even in the case of real scalars.

Throughout this section the scalars can be either $\mathbb{R}$ or $\mathbb{C}$ unless explicitly stated otherwise.

\section{Proposition 2.1.}

(a) If $X$ has the 2-dimensional 2-summing property, then $X$ is finite dimensional.

(b) If $X$ has the $k$-dimensional 2-summing property for some $k$, then so does $X^{*}$. 
(c) If $X$ has the 2-summing property, then $X$ is a maximal distance space.

Proof. For (a), we use the fact that $\ell_{1}^{m}$ fails the 2-dimensional 2-summing property for some integer $m$. In fact, in the real case, $m$ can be taken to be 3 (Example 2.3), while in the complex case, $m=4$ suffices (remark after example 2.3). Alternatively, one can check that a quotient mapping from $\ell_{1}$ onto $\ell_{2}^{2}$ has 2-summing norm larger than one, which implies that $\ell_{1}^{m}$ fails the 2-dimensional 2-summing property if $m$ is sufficiently large. So fix a norm one operator $u$ from $\ell_{1}^{m}$ into $\ell_{2}^{2}$ for which $\pi_{2}(u)>1$. By Dvoretzky's theorem, $\ell_{2}^{2}$ is almost a quotient of every infinite-dimensional space, so if $\operatorname{dim} X$ is sufficiently large, then there is an operator $Q$ from $X$ into $\ell_{2}^{2}$ with Ball $\left(\ell_{2}^{2}\right) \subset Q[\operatorname{Ball}(X)]$ but $\|Q\|<\pi_{2}(u)$. Pick $z_{1}, \ldots, z_{m}$ in Ball $(X)$ with $Q z_{i}=u e_{i}$ for $i=$ $1,2, \ldots, m$ and define $T$ from $\ell_{1}^{m}$ into $X$ by $T e_{i}=z_{i}, i=1,2, \ldots, m$. Then $u=Q T$ and $\pi_{2}(u) \leq \pi_{2}(Q)$ but $\|Q\|<\pi_{2}(u)$.

For (b), assume that $X$ has the $k$-dimensional 2 -summing property and let $T$ be any norm one operator from $X^{*}$ into $\ell_{2}^{k}$. It is enough to show that $\pi_{2}(T u) \leq 1$ when $u$ is a norm one operator from $\ell_{2}^{k}$ into $X^{*}$. This brings us back to the familiar setting of Hilbert-Schmidt operators:

$$
\pi_{2}(T u)=\pi_{2}\left(u^{*} T^{*}\right) \leq\|T\| \pi_{2}\left(u^{*}\right)=\|T\|\left\|u^{*}\right\|=1 ;
$$

the last equality following from the hypothesis that $X$ has the $k$-dimensional 2-summing property and the fact that, by (a), $X$ is reflexive.

For (c), let $T: X \rightarrow \ell_{2}^{n}$ be such that $\|T\|\left\|T^{-1}\right\|=d\left(X, \ell_{2}^{n}\right)$; then $\sqrt{n}=$ $\pi_{2}\left(T^{-1} T\right) \leq\left\|T^{-1}\right\| \pi_{2}(T)=\left\|T^{-1}\right\|\|T\|=d\left(X, \stackrel{\ell}{n}_{n}^{2}\right) \leq \sqrt{n}$. Therefore, $d\left(X, \ell_{2}^{n}\right)$ $=\sqrt{n}$.

Remark. To make the proof of (b) as simple as possible, we used (a) to reduce to the case of reflexive spaces. Actually, it is well known that if $\pi_{2}(T) \leq C\|T\|$ for every operator from $X$ into a Hilbert space $H$, then $X^{* *}$ has the same property. Indeed, it is easy to see that it is enough to consider finite rank operators from $X^{* *}$ into $H$ and then use local reflexivity (see [LT, p. 33]) and a weak* approximation argument.

Example 2.2. $\ell_{\infty}^{2}$ has the 2-summing property.

Proof. Let $u: \ell_{\infty}^{2} \rightarrow \ell_{2}^{2},\|u\|=1$. We can assume that

$$
u=\left(\begin{array}{ll}
a & b \\
0 & d
\end{array}\right)
$$

and that $(|a|+|b|)^{2}+|d|^{2} \leq 1$; or equivalently, $|a|^{2}+|b|^{2}+|d|^{2}+2|a b| \leq 1$.

For $x=\left(c_{1}, c_{2}\right) \in \ell_{\infty}^{2}$ we have that

$$
\begin{aligned}
\|u x\|^{2} & =\left|a c_{1}\right|^{2}+\left(|b|^{2}+|d|^{2}\right)\left|c_{2}\right|^{2}+2 \Re\left(a c_{1} \overline{b c_{2}}\right) \\
& \leq\left|a c_{1}\right|^{2}+\left(|b|^{2}+|d|^{2}\right)\left|c_{2}\right|^{2}+|a||b|\left(\left|c_{1}\right|^{2}+\left|c_{2}\right|^{2}\right) .
\end{aligned}
$$

Set $\lambda=|a|^{2}+|a b|$, so that $1-\lambda \geq\left(|b|^{2}+|d|^{2}\right)+|a b|$. Thus (2.1) gives

$$
\|u x\|^{2} \leq\left|c_{1}\right|^{2} \lambda+\left|c_{2}\right|^{2}(1-\lambda) \text {. }
$$

Then since (2.2) is in the form of (1.3) with constant 1 , we get that $\pi_{2}(u) \leq 1$.

Remark. At least in the complex case, Example 2.2 follows from the fact that $\alpha\left(\ell_{\infty}^{2}\right)=1$, but we thought it desirable to give a direct proof. Another proof is given in [BM]. 
Proposition 2.6 provides a useful criterion for determining whether a space has the 2-summing property. All of the intuition behind Proposition 2.6 is already contained in Example 2.3.

Example 2.3. Real $\ell_{\infty}^{3}$ and complex $\ell_{\infty}^{4}$ do not have the 2-dimensional 2summing property.

Proof. Let $x_{1}=\left(1,0, \frac{1}{\sqrt{2}}\right) ; x_{2}=\left(0,1, \frac{1}{\sqrt{2}}\right)$ and $X=\operatorname{span}\left\{x_{1}, x_{2}\right\}$ in real $\ell_{\infty}^{3}$. We denote $X$ by $X_{\infty}$ when considered as a subspace of $L_{\infty}^{3}$ and by $X_{2}$ when considered as a subspace of $L_{2}^{3}$. Also denote by $I_{\infty, 2}^{X}$ the restriction to $X$ of the identity $I_{\infty, 2}$ from $L_{\infty}^{3}$ to $L_{2}^{3}$ (we use the standard convention $L_{p}^{n}=L_{p}^{n}(\mu)$ where $\mu$ is the probability space assigning mass $\frac{1}{n}$ to every point).

We claim that $\left\|I_{\infty, 2}^{X}\right\|<1$ and that $\pi_{2}\left(I_{\infty, 2}^{X}\right)=1$.

For every $\|x\|_{\infty}=1,\left\|I_{\infty, 2} x\right\|_{2} \leq 1$ and we have equality if and only if $|x|$ is flat; i.e., $x$ is an extreme point of the unit ball of $L_{\infty}^{3}$. Then we verify that $\left\|I_{\infty, 2}^{X}\right\|<1$ by checking that $X$ does not contain any one of those vectors. For the second one, define $v: \ell_{2}^{2} \rightarrow X_{\infty}$ by $v e_{i}=x_{i}$ for $i=1,2$. Then notice that $\|v\|^{2}=\left\|\left|x_{1}\right|^{2}+\left|x_{2}\right|^{2}\right\|_{\infty}=1$, where $\left|x_{1}\right|^{2}+\left|x_{2}\right|^{2}$ is taken coordinatewise in $L_{\infty}^{3}$, and $\pi_{2}\left(I_{\infty, 2}^{X}\right)^{2} \geq \pi_{2}\left(I_{\infty, 2}^{X} v\right)^{2}=\left\|x_{1}\right\|_{2}^{2}+\left\|x_{2}\right\|_{2}^{2}=1$. The equality follows, since $\pi_{2}\left(I_{\infty, 2}^{X}\right) \leq \pi_{2}\left(I_{\infty, 2}\right)=1$. We have thus proved that $X$ does not have the 2-dimensional 2-summing property.

To conclude, define $u: L_{\infty}^{3} \rightarrow X_{2}$ by $u=P I_{\infty, 2}$, where $P$ is the orthogonal projection from $L_{2}^{3}$ onto $X_{2}$. We claim that $\|u\|<1$ and that $\pi_{2}(u)=1$.

If $\|x\|_{\infty}=1$, then $\left\|I_{\infty, 2} x\right\|_{2}=1$ iff $x$ is flat, and $\|P x\|_{2}=\|x\|_{2}$ iff $x \in X$. Since these conditions are mutually exclusive we conclude that $\|u\|<1$. But $1=\|P\|^{2} \pi_{2}\left(I_{\infty, 2}\right)^{2} \geq \pi_{2}(u)^{2} \geq \pi_{2}(u v)^{2}=\left\|P x_{1}\right\|_{2}^{2}+\left\|P x_{2}\right\|_{2}^{2}=1$.

The proof for complex $\ell_{\infty}^{4}$ is similar: Let $x_{1}=\left(1,0, \frac{1}{\sqrt{2}}, \frac{1}{\sqrt{2}}\right), x_{2}=$ $\left(1,0, \frac{i}{\sqrt{2}}, \frac{1}{\sqrt{2}}\right)$ and $X=\operatorname{span}\left\{x_{1}, x_{2}\right\}$. It is easily checked that $X$ does not contain any flat vectors and that $\left|x_{1}\right|^{2}+\left|x_{2}\right|^{2} \equiv 1$ coordinatewise.

Remark. We shall see in section 4 that complex $\ell_{\infty}^{3}$ has the 2-summing property.

Proposition 2.4. Let $X$ be an $n$-dimensional subspace of $C(K), K$ compact; $u: X \rightarrow \ell_{2}^{k}$ a map satisfying $\pi_{2}(u)=1$ and $v: \ell_{2}^{k} \rightarrow X$ satisfying $\|v\|=1$ and $\pi_{2}(u)=\pi_{2}(u v)$. Pietsch's factorization theorem gives the following diagram for some probability $\mu$ on $K$ and some norm one operator $\alpha: X_{2} \rightarrow \ell_{2}^{k}$ :

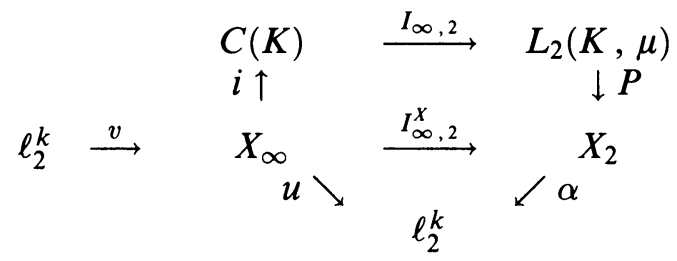

Let $Y=v\left(\ell_{2}^{k}\right)$. Then $\alpha$ is an isometry on $Y_{2}$. 
Proof. We have

$$
\begin{aligned}
1 & =\pi_{2}(u v)^{2}=\sum_{j=1}^{k}\left\|u v e_{j}\right\|^{2}=\sum_{j=1}^{k}\left\|\alpha I_{\infty, 2} v e_{j}\right\|^{2} \\
& \leq \sum_{j=1}^{k}\left\|I_{\infty, 2} v e_{j}\right\|^{2}\|\alpha\|^{2} \leq \pi_{2}\left(I_{\infty, 2}\right)^{2}=1,
\end{aligned}
$$

so $\left\|\alpha I_{\infty, 2} v e_{j}\right\|=\left\|I_{\infty, 2} v e_{j}\right\|$ for each $1 \leq j \leq k$. Recalling the elementary fact that if $S$ is an operator between Hilbert spaces, then $\{x:\|S x\|=\|S\|\|x\|\}$ is a linear subspace of the domain of $S$, we conclude that $\alpha$ is an isometry on $Y_{2}$.

Remark. Let $X$ be an $n$-dimensional space; $v: \ell_{2}^{n} \rightarrow X$ the maximum volume ellipsoid map and set $u=\frac{1}{\sqrt{n}} v^{-1}$. It is well known that $\pi_{2}(u)=1$, and since $\pi_{2}(u v)=\frac{1}{\sqrt{n}} \pi_{2}(I)=1$ we conclude that $\alpha: X_{2} \rightarrow \ell_{2}^{n}$ is an isometry. Moreover, if $X$ is of maximal distance, $u$ is the minimal volume ellipsoid map (see Remark 1.2).

Corollary 2.5. Let $X$ be an $n$-dimensional subspace of $C(K), K$ compact, and $u: X \rightarrow \ell_{2}^{n}$ be an onto map satisfying $\pi_{2}(u)=1$. Suppose that for every orthogonal projection $P$ from $\ell_{2}^{n}$ onto a proper subspace we have $\pi_{2}(P u)<1$. Then $\alpha: X_{2} \rightarrow \ell_{2}^{n}$ is an isometry ( $\alpha$ is the map appearing in Proposition 2.4). Proof. Let $v: \ell_{2}^{n} \rightarrow X_{\infty}$ be such that $\|v\|=1$ and $\pi_{2}(u)=\pi_{2}(u v)$ and let $P$ be the orthogonal projection from $\ell_{2}^{n}$ onto $u\left(v\left(\ell_{2}^{n}\right)\right)=\alpha\left(Y_{2}\right)$. We clearly have that $\pi_{2}(P u) \geq \pi_{2}(P u v)=\pi_{2}(u v)=1$. Hence, the range of $P$ cannot be a proper subspace of $X_{2}$ and therefore $\alpha$ is an isometry on $X_{2}$.

In the next proposition interpret $\frac{0}{0}$ as 0 .

Proposition 2.6. Let $X$ be an $n$-dimensional subspace of $C(K), K$ compact, and $k \leq n$. Then

$$
\begin{array}{r}
\sup \left\{\frac{\pi_{2}(u)}{\|u\|}: u: X_{\infty} \rightarrow \ell_{2}^{k}\right\}=\sup \left\{\frac{1}{\left\|P I_{\infty, 2}^{X}\right\|}: \mu \in \mathscr{P}(K), \quad P^{2}=P,\right. \\
\left.\|P\|=1, \text { rk } P \leq k, \pi_{2}\left(P I_{\infty, 2}^{X}\right)=1\right\},
\end{array}
$$

where $\mathscr{P}(K)$ consists of all the probability measures on $K$, and $I_{\infty, 2}$ is the canonical identity from $C(K)$ to $L_{2}(K, \mu)$.

Proof. It is clear that the left-hand side dominates the right one. To prove the other inequality let $u: X_{\infty} \rightarrow \ell_{2}^{k}$ be such that $\pi_{2}(u)=1$. Then find $v: \ell_{2}^{k} \rightarrow$ $X_{\infty}$ such that $\|v\|=1$ and $\pi_{2}(u v)=1$. Let $Q$ be the orthogonal projection from $\ell_{2}^{k}$ onto $u v\left(\ell_{2}^{k}\right)=\alpha\left(Y_{2}\right)$ (with the notation of Proposition 2.4), and $P$ be the orthogonal projection from $X_{2}$ onto $Y_{2}$. Notice that $Q u=\alpha P I_{\infty, 2}^{X}$. Since $\alpha$ is an isometry on $Y_{2}$ we have that $\pi_{2}\left(P I_{\infty, 2}^{X}\right)=\pi_{2}(Q u)=1$ and that $\left\|P I_{\infty, 2}^{X}\right\|=\|Q u\| \leq\|u\|$. Therefore

$$
\frac{1}{\left\|P I_{\infty, 2}^{X}\right\|} \geq \frac{\pi_{2}(u)}{\|u\|}
$$


Proposition 2.6 has a nice form when $X$ is 2-dimensional because then we do not need to take the orthogonal projection on $X_{2}$. Indeed, if $P$ has rank one then it is clear that $\pi_{2}\left(P I_{\infty, 2}^{X}\right)=\left\|P I_{\infty, 2}^{X}\right\|$. If $\mu$ is a probability measure on $K$ with support $K_{0}$, then $\left\|I_{\infty, 2}^{X}\right\|<1$ iff Ball $(X)$ does not contain any "flat" vector on $K_{0}$; i.e., whenever $x \in X$ and $\|x\|=1$, then we have that $|x|_{\mid K_{0}} \not \equiv 1$. On the other hand, $\pi_{2}\left(I_{\infty, 2}^{X}\right)=1$ iff there exist vectors $x_{1}, x_{2}$ in $X$ such that $\left|x_{1}\right|^{2}+\left|x_{2}\right|^{2} \leq 1$ on $K$ and $\left|x_{1}\right|^{2}+\left|x_{2}\right|^{2} \equiv 1$ on $K_{0}$. To see why the second statement is true, find $v: \ell_{2}^{2} \rightarrow X_{\infty}$ satisfying $\|v\|=1$ and $\pi_{2}\left(I_{\infty, 2}^{X} v\right)=1$. Then let $x_{i}=v e_{i}$ for $i=1,2$ and the result is easily checked for these vectors. Since every closed subset of a compact metric space is the support of some probability measure, this discussion proves:

Corollary 2.7.a. Let $X$ be a 2-dimensional subspace of $C(K), K$ compact metric. Then $X$ does not have the 2-summing property if and only if there exist vectors $x_{1}, x_{2}$ in $X$ and a closed set $K_{0} \subset K$ with $1_{K_{0}} \leq\left|x_{1}\right|^{2}+\left|x_{2}\right|^{2} \leq 1$ such that for every $x \in X$ with $\|x\|_{\infty}=1$, we have that $|x|_{\mid K_{0}} \not \equiv 1$.

If $X \subset C(K)$ contains a vector $|x| \equiv 1$, then for every probability measure $\mu$ on $K$ we have $\left\|I_{\infty, 2} x\right\|=\|x\|$; hence, $\left\|I_{\infty, 2}^{X}\right\|=1$ and we have

Corollary 2.8. Let $X$ be a 2-dimensional subspace of $C(K), K$ compact. If $X$ contains a vector $x,|x| \equiv 1$ on $K$ then $X$ has the 2-summing property.

This applies immediately to $\ell_{\infty}^{2}$ (both real and complex) and also to $\ell_{1}^{2}$ (again real and complex) if embedded in a canonical way. It also implies that there are a continuum of pairwise nonisometric two dimensional complex spaces which have the 2-summing property. We shall see later that the real 2-summing property is quite different from the complex version. For the moment, take $X=\operatorname{span}\left\{\left(1,0, \frac{1}{\sqrt{2}}\right),\left(0,1, \frac{1}{\sqrt{2}}\right)\right\}$ inside $\ell_{\infty}^{3}$. We proved in Example 2.3 that real $X$ does not have the 2-summing property. However, complex $X$ has it. To see this, notice that $\left(1,0, \frac{1}{\sqrt{2}}\right)+i\left(0,1, \frac{1}{\sqrt{2}}\right)$ is "flat" and hence Corollary 2.8 implies the result. The difference can be explained by saying that it is easier to get "flat" vectors in the complex setting (see Proposition 4.4).

Let us return to the discussion following Proposition 2.6. Suppose that $X$ is an $n$-dimensional subspace of $C(K), \mu$ is a probability measure on $K$ with support $K_{0} \subset K$, and $P$ is an orthogonal projection from $X_{2} \subset L_{2}(\mu)$ onto a subspace $Y_{2}$. Notice that $\pi_{2}\left(P I_{\infty, 2}^{X}\right)=1$ if and only if there exist vectors $x_{1}, x_{2}, \ldots, x_{n}$ in $Y$ with $1_{K_{0}} \leq \sum_{j=1}^{n}\left|x_{j}\right|^{2} \leq 1$ and each vector $I_{\infty, 2} x_{j}$ is in $Y_{2}$. (Keep in mind that $Y_{2}$ is relatively $L_{2}(\mu)$-closed in $X_{2}$, hence if $y \in Y$, $z \in X$, and $1_{K_{0}} y=1_{K_{0}} z$, then also $z$ is in $Y$.) Similarly, $\left\|P I_{\infty, 2}^{X}\right\|=1$ if and only if there exists a single vector $x$ in $Y$ with $1_{K_{0}} \leq|x| \leq 1$. Thus we get a version of Corollary 2.7.a for all finite-dimensional spaces:

Corollary 2.7.b. Let $X$ be a finite-dimensional subspace of $C(K), K$ compact metric. Then $X$ does not have the 2-summing property if and only if there exist vectors $x_{1}, x_{2}, \ldots, x_{n}$ in $X$ and a closed set $K_{0} \subset K$ with $1_{K_{0}} \leq \sum_{j=1}^{n}\left|x_{j}\right|^{2} \leq$ 1 such that for every $x \in X$ with $1_{K_{0}} \leq|x| \leq 1$, we have that $1_{K_{0}} x$ is not in $\operatorname{span}\left\{1_{K_{0}} x_{1}, 1_{K_{0}} x_{2}, \ldots, 1_{K_{0}} x_{n}\right\}$.

In the complex case, the 2-summing property is not hereditary, since complex $\ell_{1}^{3}$ has the 2-summing property but $\ell_{1}^{2}$ is the only 2-dimensional subspace of 
it which has the 2-summing property (see Theorem 4.2 and Proposition 5.7.) Nevertheless:

Proposition 2.9. Let $X$ be a subspace of $\ell_{\infty}^{N}$ which has the 2-summing property. Then every subspace of $X$ has the 2-summing property.

Proof. Assume that $X$ has a subspace which fails the 2-summing property. Write $K=\{1,2, \ldots, N\}$. Since $\ell_{\infty}^{N}=C(K)$, we can apply Corollary 2.7.b. There exists a subset $K_{0} \subset K$ for which we can find vectors $x_{1}, x_{2}, \ldots, x_{n}$ in $X$ with $1_{K_{0}} \leq \sum_{j=1}^{n}\left|x_{j}\right|^{2} \leq 1$ such that no norm one vector in $Y \equiv$ span $\left\{x_{1}, \ldots, x_{n}\right\}$ is unimodular on $K_{0}$. We can also assume that $K_{0}$ is maximal with respect to this property; in particular, $\sum_{j=1}^{n}\left|x_{j}\right|^{2}$ is strictly less than one off $K_{0}$ and hence $\sum_{j=1}^{n} 1_{\sim K_{0}}\left|x_{j}\right|^{2}<1-\epsilon$ for some $\epsilon>0$.

On the other hand, since $X$ has the 2-summing property, there exists a vector $y \in Y$ which is unimodular on $K_{0}$ (and whose restriction to $K_{0}$ agrees with the restriction to $K_{0}$ of some unit vector in $\left.X\right)$. Evidently $\|y\|>1$. Thus there exists $1>\tau>0$ so that $z=z_{\tau} \equiv\left|\tau^{\frac{1}{2}} y\right|^{2}+\sum_{j=1}^{n}\left|(1-\tau)^{\frac{1}{2}} x_{j}\right|^{2}$ satisfies $\left\|1_{\sim K_{0}} z\right\|_{\infty}=1$. But then $z \leq 1$, a square function of a system from $Y$, is unimodular on a set which properly contains $K_{0}$; this contradicts the maximality of $K_{0}$.

For any real Banach space $F$, let $F_{\mathbb{C}}$ denote the linear space $F \oplus F$ with complex structure defined by means of the formula $(a+b i)\left(f_{1} \oplus f_{2}\right)=\left(a f_{1}-\right.$ $\left.b f_{2}\right) \oplus\left(a f_{2}+b f_{1}\right)$. There is a natural topology on $F_{\mathbb{C}}$, namely $F_{\mathbb{C}}$ is homeomorphic with the direct sum of two real Banach spaces $F \oplus F$. In two special cases we shall define a norm on $F_{\mathbb{C}}$ which will make it a complex Banach space. First, if $E$ is a linear subspace of some real $\ell_{\infty}^{k}$, we endow $E_{\mathbb{C}}$ with the norm induced from complex $\ell_{\infty}^{k}$ by means of the obvious embedding. Secondly, if $E=H$ is a Hilbert space, then $H_{\mathbb{C}}$ is normed by means of the formula $\left\|h_{1} \oplus h_{2}\right\|=\left(\left\|h_{1}\right\|^{2}+\left\|h_{2}\right\|^{2}\right)^{1 / 2}$. These two definitions are consistent, because $H$ is isometric to a subspace of real $\ell_{\infty}^{k}$ only if $\operatorname{dim}_{\mathbb{R}} H \leq 1$. Now, if $T: F \rightarrow G$ is a linear operator, we define $T_{\mathbb{C}}: F_{\mathbb{C}} \rightarrow G_{\mathbb{C}}$ by the formula $T_{\mathbb{C}}\left(f_{1} \oplus f_{2}\right)=\left(T f_{1} \oplus T f_{2}\right)$.

Proposition 2.10. Let $E$ be a subspace of real $\ell_{\infty}^{k}$ and $S: E \rightarrow \ell_{2}^{2}$ be a reallinear mapping from $E$ into a 2-dimensional real Hilbert space. Then

$$
\pi_{2}\left(S_{\mathbb{C}}\right)=\pi_{2}(S)=\left\|S_{\mathbb{C}}\right\| \text {. }
$$

Proof. From the discussion in section 1 we see that there are vectors $x, y$ in $E$ such that $|x|^{2}+|y|^{2} \leq 1$ (interpreted coordinatewise) and

$$
\pi_{2}(S)=\left(\|S x\|^{2}+\|S y\|^{2}\right)^{1 / 2} \text {. }
$$

Observe that, by our definition, $\|x \oplus y\|_{E_{\mathrm{c}}}=\|x+i y\|_{l_{\infty}^{k}(\mathbb{C})} \leq 1$. It follows that

$$
\pi_{2}\left(S_{\mathbb{C}}\right) \geq\left\|S_{\mathbb{C}}\right\| \geq\left\|S_{\mathbb{C}}(x \oplus y)\right\|_{H_{\mathbb{C}}}=\left(\|S x\|^{2}+\|S y\|^{2}\right)^{1 / 2}=\pi_{2}(S),
$$

hence it remains to verify that $\pi_{2}\left(S_{\mathbb{C}}\right) \leq \pi_{2}(S)$.

Take $u, w$ in $E_{\mathbb{C}}$ with $|u|^{2}+|w|^{2} \leq 1$ and $\pi_{2}\left(S_{\mathbb{C}}\right)^{2}=\left\|S_{\mathbb{C}}(u)\right\|^{2}+\left\|S_{\mathbb{C}}(w)\right\|^{2}$. Interpreting real and imaginary parts of vectors in $\ell_{\infty}^{k}$ coordinatewise, we see that $\Re u, \Im u$ are in $E$ and similarly for $w$. Moreover,

$$
\left\|S_{\mathbb{C}}(u)\right\|^{2}+\left\|S_{\mathbb{C}}(w)\right\|^{2}=\|S \Re u\|^{2}+\|S \Im u\|^{2}+\|S \Re w\|^{2}+\|S \Im w\|^{2} .
$$


This last quantity is at most $\pi_{2}(S)$ since $|\Re u|^{2}+|\Im u|^{2}+|\Re w|^{2}+|\Im w|^{2}=$ $|u|^{2}+|w|^{2} \leq 1$.

It is easy to determine when a complex-linear operator is the complexification of a real-linear operator:

Proposition 2.11. Let $E$ be a real Banach space and let $G$ be a complex Hilbert space. Let $T: E_{\mathbb{C}} \rightarrow G$ be a complex-linear continuous operator. The following conditions are equivalent:

(i) There is a real Hilbert space $H$ and continuous linear operators $S$ : $E \rightarrow H, U: H_{\mathbb{C}} \rightarrow G$, such that $T=U \circ S_{\mathbb{C}}$ and $U$ is an isometric embedding.

(ii) For each $e, e^{\prime} \in E$ one has $\left\|T\left(e+i e^{\prime}\right)\right\|=\left\|T\left(e-i e^{\prime}\right)\right\|$.

(iii) For each $e, e^{\prime} \in E$ one has $\Im\left(T e, T e^{\prime}\right)=0$.

(iv) There is a subset $E_{0}$ of $E$ such that the linear span of $E_{0}$ is dense in $E$ and for each $e, e^{\prime}$ in $E_{0}, \Im\left(T e, T e^{\prime}\right)=0$.

Proof. (i) implies (ii), because

$$
\left\|T\left(e \pm i e^{\prime}\right)\right\|=\left\|S_{\mathbb{C}}\left(e \pm i e^{\prime}\right)\right\|=\left(\|S e\|^{2}+\left\|S e^{\prime}\right\|^{2}\right)^{1 / 2} .
$$

To see that (ii) implies (i) we let $H$ denote the closure of $T(E)$ in $G$. Observe that, if $x=e \oplus e^{\prime} \in E_{\mathbb{C}}$, then using the parallelogram identity we obtain

$$
\left\|T e+i T e^{\prime}\right\|^{2}=\frac{1}{2}\left(\left\|T e+i T e^{\prime}\right\|^{2}+\left\|T e-i T e^{\prime}\right\|^{2}\right)=\|T e\|^{2}+\left\|T e^{\prime}\right\|^{2} .
$$

Hence $H \oplus i H$ is linearly isometric to $H_{\mathbb{C}}$ and, if $U$ denotes the natural embedding and $S=\left.T\right|_{E}$, then we have $T=U \circ S_{\mathbb{C}}$.

It is clear that (iii) and (iv) are equivalent. On the other hand, the identity

$$
\begin{aligned}
& \left\|T\left(e+i e^{\prime}\right)\right\|^{2}-\left\|T\left(e-i e^{\prime}\right)\right\|^{2} \\
& \quad=\left(T e+i T e^{\prime}, T e+i T e^{\prime}\right)-\left(T e-i T e^{\prime}, T e-i T e^{\prime}\right) \\
& \quad=2 i\left(\left(T e^{\prime}, T e\right)-\left(T e, T e^{\prime}\right)\right)=4 \Im\left(T e, T e^{\prime}\right),
\end{aligned}
$$

makes it obvious that (ii) and (iii) are equivalent.

\section{THE REAL CASE}

Throughout this section we deal with spaces over the reals. Example 2.3 and Proposition 2.1 imply that there are many norm one operators from real $\ell_{1}^{3}$ into $\ell_{2}^{2}$ whose 2 -summing norm is larger than one.

Proposition 3.1. Let $u$ be an operator from real $\ell_{1}^{3}$ to $\ell_{2}^{2}$ such that $u e_{1}, u e_{2}, u e_{3}$ have norm one and every two of them are linearly independent. Then $\pi_{2}(u)>1$. Proof. Let $u: \ell_{1}^{3} \rightarrow \ell_{2}^{2}$ satisfy the assumptions of Proposition 3.1, and set $x_{i}=u e_{i}, i=1,2,3$. Notice that $\ell_{1}^{3}$ embeds isometrically via the natural evaluation mapping into $C(K)$, where $K=\{(1,1,1),(1,1,-1),(1,-1,1)$, $(-1,1,1)\}$, regarded as a subset of $\ell_{\infty}^{3}=\left(\ell_{1}^{3}\right)^{*}$. Assume that $\pi_{2}(u)=1$ and consider the Pietsch factorization diagram: 


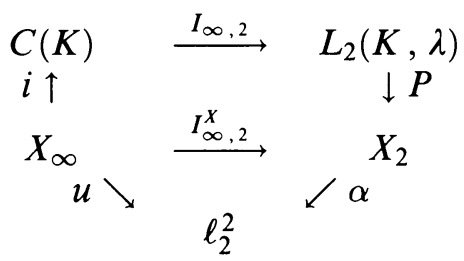

So $\|\alpha\|=\pi_{2}(u)=1$. We claim that $\operatorname{dim} X_{2}=3$. (This is not automatic since the support $K_{0}$ of $\lambda$ may not be all of $K$.) Indeed, any two of $\left\{f_{1}, f_{2}, f_{3}\right\}$ are linearly independent, since this is true of $\left\{\alpha f_{1}, \alpha f_{2}, \alpha f_{3}\right\}$, so the cardinality of $K_{0}$ is at least three. But $\left\{f_{i \mid L}\right\}_{i=1}^{3}$ is linearly independent if $L$ is any three (or four) element subset of $K$.

To complete the proof, just recall that no norm one linear operator $\beta$ from $\ell_{2}^{3}$ into $\ell_{2}^{2}$ achieves its norm at three linearly independent vectors since, e.g., the set $\left\{x \in \ell_{2}^{3}:\|\beta x\|=\|\beta\|\|x\|\right\}$ is a subspace of $\ell_{2}^{3}$.

Having treated the "worst" case of $\ell_{1}^{n}$, it is easy to formulate a version of Proposition 3.1 for general spaces.

Corollary 3.2. Let $X$ be a real space.

(a) If $T$ is a norm one operator from $X$ into $\ell_{2}^{2}$ such that for some $z_{1}, z_{2}$, $z_{3}$ in Ball $(X), T z_{1}, T z_{2}, T z_{3}$ have norm one and every two of them are linearly independent, then $\pi_{2}(T)>1$.

(b) If there exist $u: \ell_{2}^{2} \rightarrow X$ and $x_{1}, x_{2}, x_{3} \in$ Ball $\left(\ell_{2}^{2}\right)$ such that $\|u\|=$ $1=\left\|u x_{i}\right\|=1$ for $i=1,2,3$ and every two of $x_{1}, x_{2}, x_{3}$ are linearly independent, then $X$ does not have the 2-dimensional 2-summing property.

Proof. For (a), define $w: \ell_{1}^{3} \rightarrow X$ by $w e_{i}=z_{i}, i \leq 3$. Then $T w$ satisfies the hypothesis of Proposition 3.1. Therefore $\pi_{2}(T w)>1$; and since $\|w\|=1$ we have that $\pi_{2}(T)>1$.

For (b) it is enough to prove that $X^{*}$ does not have the 2-dimensional 2summing property. If $x_{i}^{*} \in$ Ball $\left(X^{*}\right), i=1,2,3$, satisfy $\left\langle x_{i}^{*}, u x_{i}\right\rangle=1$, then $1 \geq\left\|u^{*} x_{i}^{*}\right\| \geq\left\langle u^{*} x_{i}^{*}, x_{i}\right\rangle=\left\langle x_{i}^{*}, u x_{i}\right\rangle=1$; therefore, $u^{*} x_{i}^{*}=x_{i}$ for $i=1,2,3$ and the previous part gives us that $\pi_{2}\left(u^{*}\right)>1$.

We are now ready for the main result of this section:

Theorem 3.3. If $X$ is a real space of dimension at least three, then $X$ does not have the 2-summing property. Consequently, the only real spaces which have the 2-summing property are $\mathbb{R}$ and $\ell_{\infty}^{2}$.

Proof. The "consequently" follows from the first statement and Proposition 2.1 (c) because in the real case $\ell_{\infty}^{2}$ is the only 2-dimensional maximal distance space. This is an unpublished result of Davis and the second author; for a proof see Lewis' paper [L].

So assume that $X$ is $\mathbb{R}^{n}, n \geq 3$, under some norm and has the 2-summing property. We can also assume that the usual Euclidean ball $\mathscr{E}$ is the ellipsoid of minimal volume containing Ball $(X)$, and we use $|\cdot|$ to denote the Euclidean norm.

By John's theorem, there exist $\mu_{1}, \ldots, \mu_{m}>0$ such that $\sum_{i=1}^{m} \mu_{i}=n$ and $y_{1}, \ldots, y_{m} \in X$ outside contact points (i.e., $\left\|y_{i}\right\|=\left|y_{i}\right|=1$ for $i=$ 
$1,2, \ldots, m)$ such that every $x \in X$ satisfies $x=\sum_{i=1}^{m} \mu_{i}\left\langle x, y_{i}\right\rangle y_{i}$. Recall that $\mathscr{E} / \sqrt{n} \subset$ Ball $(X)$, in fact, by Lemma $1.1, \mathscr{E} / \sqrt{n}$ is the ellipsoid of maximal volume contained in Ball $(X)$.

If $x$ is an inside contact point (i.e., $\|x\|=1$ and $|x|=1 / \sqrt{n}$ ), Milman and Wolfson $[\mathrm{MW}]$ proved that

$$
\left|\left\langle x, y_{i}\right\rangle\right|=\frac{1}{n} \quad \text { for every } i=1,2, \ldots, m .
$$

To see this, observe that $\{z \in X:\langle z, x\rangle=1 / n\}$ supports $\mathscr{E} / \sqrt{n}$ at the inside contact point $x$, hence-draw a picture-the norm of $\langle\cdot, x\rangle$ in $X^{*}$ is $1 / n$. Thus

$$
\frac{1}{n}=\langle x, x\rangle=\sum_{i=1}^{m} \mu_{i}\left\langle x, y_{i}\right\rangle^{2} \leq \sum_{i=1}^{m} \mu_{i}\|\langle\cdot, x\rangle\|_{X^{*}}^{2}\left\|y_{i}\right\|^{2}=\frac{1}{n} .
$$

This implies that $\left|\left\langle x, y_{i}\right\rangle\right|=\frac{1}{n}$ for $i=1,2, \ldots, m$ and proves (3.1).

In other words, the norm one (in $X^{*}$ ) functional $\langle\cdot, n x\rangle$ norms all of the $y_{i}$ 's as well as $x$. This implies that both conv $\left\{y_{i}:\left\langle y_{i}, n x\right\rangle=1\right\}$ and conv $\left\{y_{i}\right.$ : $\left.\left\langle y_{i}, n x\right\rangle=-1\right\}$ are subsets of the unit sphere of $X$. Now we know that $X$ has maximal distance, hence at least one inside contact point exists, whence Ball $(X)$ has at least two "flat" faces.

The next step is to observe that we can find $n$ linearly independent outside contact points $y_{1}, \ldots, y_{n}$ and $n$ linearly independent inside contact points $x_{1}, \ldots, x_{n}$ satisfying (3.1). This will give us enough faces on Ball $(X)$ so that a 2-dimensional section will be a hexagon and we can apply Corollary 3.2 (b). Now the John representation of the identity gives the existence of the outside contact points, and since $\mathscr{E} / \sqrt{n}$ is the ellipsoid of maximal volume contained in Ball $(X)$, another application of John's theorem gives the inside coniact points.

So let $y_{1}, \ldots, y_{n}$ be linearly independent outside contact points, and let $x_{1}, \ldots, x_{n}$ linearly independent inside contact points satisfying (3.1), such that for the first three of them we have

$$
\begin{array}{lll}
\left\langle n x_{1}, y_{1}\right\rangle=1, & \left\langle n x_{1}, y_{2}\right\rangle=1, & \left\langle n x_{1}, y_{3}\right\rangle=1, \\
\left\langle n x_{2}, y_{1}\right\rangle=1, & \left\langle n x_{2}, y_{2}\right\rangle=1, & \left\langle n x_{2}, y_{3}\right\rangle=-1, \\
\left\langle n x_{3}, y_{1}\right\rangle=1, & \left\langle n x_{3}, y_{2}\right\rangle=-1, & \left\langle n x_{3}, y_{3}\right\rangle=1 .
\end{array}
$$

(We are allowed to change signs and renumber the contact points.) Let $v$ denote the linear map from span $\left\{y_{1}, y_{2}, y_{3}\right\}$ into $l_{1}^{3}$ which takes $y_{i}$ to $e_{i}$. Then $\|v y\|_{1}=\|y\|$ if $y=\sum_{1}^{3} \alpha_{i} y_{i}$ and either $\alpha_{1} \alpha_{2} \geq 0$ or $\alpha_{1} \alpha_{3} \geq 0$, hence the restriction of $v$ to $F=\operatorname{span}\left\{\frac{y_{1}+y_{2}}{2}, \frac{y_{1}+y_{3}}{2}\right\}$ is an isometry. But then $v$ [Ball $\left.(F)\right]$ is a regular hexagon, which implies that the maximum volume ellipsoid for Ball $(F)$ touches the unit sphere of $F$ at six points. We finish by applying Corollary 3.2 (b).

Lewis [L] proved that every real maximal distance space of dimension at least two contains a subspace isometrically isomorphic to $\ell_{1}^{2}$; that is, a subspace whose unit ball is a parallelogram. In view of Remark 1.2, the proof of Theorem 3.3 yields:

Corollary 3.4. If $X$ is a real maximal distance space of dimension at least three, then $X$ has a subspace whose unit ball is a regular hexagon. 


\section{THE COMPLEX CASE}

As was mentioned in the introduction, the study of the 2-summing propertyin the complex case is a priori more complicated than in the real case even for two dimensional spaces simply because in the real case there is only one 2-dimensional maximal distance space, while in the complex space there are at least two, $\ell_{\infty}^{2}$ and its dual. In fact, it is not difficult to construct other complex 2-dimensional maximal distance spaces without using Corollary 2.8. One way is to use John's representation theorem; this was done independently by Gowers and Tomczak-Jaegermann [both unpublished] a couple of years ago in order to construct real 4-dimensional maximal distance spaces whose unit ball is not strictly convex; this approach also yields maximal distance 2-dimensional complex spaces different from $\ell_{\infty}^{2}$ and $\ell_{1}^{2}$. However, a simpler way of seeing that there are many maximal distance 2-dimensional complex spaces is via Proposition 4.1:

Proposition 4.1. Suppose that $X$ is an $n$-dimensional complex space which, as a real space, contains a real $n$-dimensional subspace which has maximal distance to real $\ell_{2}^{n}$. Then $X$ has maximal distance to complex $\ell_{2}^{n}$.

Proof. Let $Y$ be a real $n$-dimensional subspace of $X$ which has maximal distance to real $\ell_{2}^{n}$, and suppose that $T$ is any complex linear isomorphism from $X$ to complex $\ell_{2}^{n}$. But considered as a real space, complex $\ell_{2}^{n}$ is just real $\ell_{2}^{2 n}$, and so the restriction of $T$ to $Y$ is a real linear isomorphism from $Y$ to a real $n$-dimensional Hilbert space, hence by the assumption on $Y$,

$$
\|T\|\left\|T^{-1}\right\| \geq\left\|T_{\mid Y}\right\|\left\|\left(T_{\mid Y}\right)^{-1}\right\| \geq \sqrt{n},
$$

so the desired conclusion follows.

Proposition 4.1 makes it easy to construct in an elementary manner 2-dimensional complex maximal distance spaces which are not isometric to either $\ell_{\infty}^{2}$ or $\ell_{1}^{2}$. For example, in complex $\ell_{\infty}^{3}$, let $x=(1,0, a)$ and $y=(0,1, b)$ with $|a+b| \vee|a-b| \leq 1$ but $|a|+|b|>1$, and set $X=\operatorname{span}\{x, y\}$. In Proposition 4.4 we prove that every 2 -dimensional subspace of complex $\ell_{\infty}^{3}$ is a maximal distance space, by proving that they all have the 2 -summing property.

However, in the complex setting, the 2-summing property is not restricted to 2-dimensional spaces. In fact, we do not know a good bound for the dimension of complex spaces which have the 2-summing property, although we suspect that dimension three is the limit. In dimension three itself, we know of only two examples, $\ell_{1}^{3}$ and its dual.

We can prove Theorem 4.2 using Proposition 2.6 and the proof of Proposition 4.4 (see Remark 4.6). The proof we give is of independent interest.

Theorem 4.2. Complex $\ell_{1}^{3}$ has the 2-summing property. Hence also $\ell_{\infty}^{3}$ has the 2-summing property.

Proof. The proof reduces the theorem to the following calculus lemma, which we prove after giving the reduction:

Lemma 4.3. Fix arbitrary complex numbers $\lambda_{1}, \lambda_{2}, \lambda_{3}$ and define a function $f$ on the bidisk by

$$
f\left(z_{1}, z_{2}\right)=\left|1+\lambda_{1} z_{1}+\lambda_{2} z_{2}+\lambda_{3} z_{1} \overline{z_{2}}\right|+\left|\lambda_{3}\right| \sqrt{1-\left|z_{1}\right|^{2}} \sqrt{1-\left|z_{2}\right|^{2}} .
$$


Then the maximum of $f$ is attained at some point on the 2-dimensional torus; that is, when $\left|z_{1}\right|=\left|z_{2}\right|=1$.

Reduction to Lemma 4.3. Let $K=\{1\} \times \mathbb{T} \times \mathbb{T}$, where $\mathbb{T}$ is the unit circle, and regard $\ell_{1}^{3}$ as the subspace of $C(K)$ spanned by the coordinate projections $f_{0}, f_{1}, f_{2}$. Let $u$ be a norm one operator from $\ell_{1}^{3}$ into $\ell_{2}$ (complex scalars). We want to show that $\pi_{2}(u)=1$. That is, we want a probability $\mu$ on $K$ so that for each $x \in \ell_{1}^{3}$,

$$
\|u x\|^{2} \leq \int|x(k)|^{2} d \mu(k) .
$$

Notice that if we add to the $u f_{j}$ 's mutually orthogonal vectors which are also orthogonal to the range of $u$, the $\pi_{2}$-norm of the resulting operator can only increase. Thus we assume, without loss of generality, that $\left\|u f_{j}\right\|=1$ for $j=$ $0,1,2$, and that

$$
u f_{0}=\delta_{0}, \quad u f_{1}=\alpha_{1} \delta_{0}+\beta_{1} \delta_{1}, \quad u f_{2}=\alpha_{2} \delta_{0}+\beta_{2} \delta_{1}+\gamma_{2} \delta_{2},
$$

where $\left\{\delta_{0}, \delta_{1}, \delta_{2}\right\}$ is an orthonormal set of $\ell_{2}$. Define a linear functional $F$ on $E=\operatorname{span}\left\{f_{0}, f_{1}, f_{2}, f_{1} \bar{f}_{2}\right\}$ by

$$
F f_{0}=1, \quad F f_{1}=\alpha_{1}, \quad F f_{2}=\alpha_{2}, \quad F\left(f_{1} \bar{f}_{2}\right)=\alpha_{1} \overline{\alpha_{2}}+\beta_{1} \bar{\beta}_{2} .
$$

Claim. $\|F\|=1$ as a linear functional on $\left(E,\|\cdot\|_{C(K)}\right)$.

Assume the claim. Then by the Hahn-Banach theorem, $F$ can be extended to a norm one linear functional, also denoted by $F$, on $C(K)$. Since $\|F\|=1=$ $F f_{0}, F$ is given by integration against a probability, say, $\mu$. Now by the definition of $F$, the mapping $v:\left\{0, f_{0}, f_{1}, f_{2}\right\} \rightarrow \ell_{2}$ defined by $v 0=0, v f_{j}=u f_{j}$ for $j=0,1,2$ is $L_{2}(\mu)$-to- $\ell_{2}$ inner product preserving, hence an $L_{2}$-isometry, whence extends to a linear isometry from $\left(\operatorname{span}\left\{f_{0}, f_{1}, f_{2}\right\},\|\cdot\|_{L_{2}(\mu)}\right)$ into $\ell_{2}$. This shows that $\pi_{2}(u) \leq 1$, as desired.

Proof of claim. The claim says that for all $\left\{\lambda_{j}\right\}_{j=0}^{3}$ in $\mathbb{C}^{4}$,

$$
\left|\lambda_{0}+\lambda_{1} \alpha_{1}+\lambda_{2} \alpha_{2}+\lambda_{3}\left(\alpha_{1} \overline{\alpha_{2}}+\beta_{1} \overline{\beta_{2}}\right)\right| \leq \sup _{\left(z_{1}, z_{2}\right) \in T \times T}\left|\lambda_{0}+\lambda_{1} z_{1}+\lambda_{2} z_{2}+\lambda_{3} z_{1} \overline{z_{2}}\right| .
$$

But the left-hand side is dominated by

$$
\left|\lambda_{0}+\lambda_{1} \alpha_{1}+\lambda_{2} \alpha_{2}+\lambda_{3} \alpha_{1} \bar{\alpha}_{2}\right|+\left|\lambda_{3}\right| \sqrt{1-\left|\alpha_{1}\right|^{2}} \sqrt{1-\left|\alpha_{2}\right|^{2}},
$$

so the claim follows from Lemma 4.3.

Proof of Lemma 4.3. For fixed $z_{2}$, you can rotate $z_{1}$ to see that at the maximum

$$
f\left(z_{1}, z_{2}\right)=\left|1+\lambda_{2} z_{2}\right|+\left|z_{1}\right|\left|\lambda_{1}+\lambda_{3} \bar{z}_{2}\right|+\left|\lambda_{3}\right| \sqrt{1-\left|z_{1}\right|^{2}} \sqrt{1-\left|z_{2}\right|^{2}} .
$$

As $\left|z_{1}\right|$ varies, $\left(\left|z_{1}\right|, \sqrt{1-\left|z_{1}\right|^{2}}\right)$ varies over the unit sphere of $\ell_{2}^{2}$, so at the maximum

$$
f\left(z_{1}, z_{2}\right)=\left|1+\lambda_{2} z_{2}\right|+\sqrt{\left|\lambda_{1}+\lambda_{3} \bar{z}_{2}\right|^{2}+\left|\lambda_{3}\right|^{2}\left(1-\left|z_{2}\right|^{2}\right)} .
$$

In particular, $\left|z_{1}\right|=1$ if and only if $\left|z_{2}\right|=1$. The last expression can be rewritten as

$$
\left|1+\lambda_{2} z_{2}\right|+\sqrt{\left|\lambda_{1}\right|^{2}+\left|\lambda_{3}\right|^{2}+2 \Re\left(\lambda_{1} \overline{\lambda_{3}} z_{2}\right)} .
$$


Choose $\theta$ so that $e^{i \theta} \lambda_{1} \overline{\lambda_{3}}$ is purely imaginary; so $\Re\left(\lambda_{1} \overline{\lambda_{3}} z_{2}\right)$ does not change if you add a real multiple of $e^{i \theta}$ to $z_{2}$. Assume first that $\lambda_{2} \neq 0$. Then, for any $\epsilon>0$, by adding either $\epsilon e^{i \theta}$ or $-\epsilon e^{i \theta}$ to $z_{2}$ you increase the first term; this means that at the maximum $\left|z_{2}\right|=1$. The case where $\lambda_{2}=0$ can be handled in a similar way.

Remarks. 1. A similar argument reduces the problem of whether $\ell_{1}^{4}$ has the 2summing propertyto a calculus problem; however, in the remark after Example 2.3 we give a simple direct argument that $\ell_{\infty}^{4}$ fails the 2-summing property.

2. Bagchi and Misra [BM] give a different reduction of Theorem 4.2 to a variation of Lemma 4.3. Their argument may be more appealing to operator theorists.

3. The following proposition is a consequence of Proposition 2.9 and Theorem 4.2. We indicate a shorter argument.

Proposition 4.4. Every 2-dimensional subspace of $\ell_{\infty}^{3}$ has the 2-summing property.

Proof. Assume that $X \subset \ell_{\infty}^{3}$ is 2-dimensional. Applying $\ell_{\infty}^{3}$ isometries we assume that $X$ has a basis of the form $\left\{\left(1,0, a_{1}\right),\left(0,1, a_{2}\right)\right\}$ where $\left|a_{i}\right| \leq 1$ for $i=1,2$. If $\left|a_{1}\right|+\left|a_{2}\right| \leq 1$ then $X$ is isometric to $\ell_{\infty}^{2}$ and by Example 2.2 it has the 2-summing property. So assume that $\left|a_{1}\right|+\left|a_{2}\right|>1$. It is easy to find $\theta, 0 \leq \theta<2 \pi$ such that $\left|a_{1}+e^{i \theta} a_{2}\right|=1$. Hence, $\left(1,0, a_{1}\right)+e^{i \theta}\left(0,1, a_{2}\right)$ is "flat" and the result follows from Corollary 2.8.

It is interesting to notice that Proposition 4.4 is equivalent to the following calculus formulation.

Proposition 4.5. Given any complex numbers $c_{1}, c_{2}, c_{3}$ and $d_{1}, d_{2}, d_{3}$ with $\left|c_{j}\right|^{2}+\left|d_{j}\right|^{2} \leq 1$ for $j=1,2,3$, suppose that $\alpha, \beta$ satisfy

$$
|\alpha|^{2}|\gamma|^{2}+|\beta|^{2}|\delta|^{2} \leq \max _{j=1,2,3}\left|\gamma c_{j}+\delta d_{j}\right|^{2} \quad \forall \gamma, \delta \in \mathbb{C} .
$$

Then $|\alpha|^{2}+|\beta|^{2} \leq 1$.

To see the equivalence of Propositions 4.4 and 4.5, let $X$ be a 2-dimensional subspace of $\ell_{\infty}^{3}$ and $X \stackrel{u}{\longrightarrow} \ell_{2}^{2}$ a norm one operator. Choose $\ell_{2}^{2} \stackrel{v}{\longrightarrow} X$ of norm one so that $\pi_{2}(u)=\pi_{2}(u v)$. We can assume that $u v$ is diagonal; say, $u v\left(e_{1}\right)=$ $\alpha e_{1}$ and $u v\left(e_{2}\right)=\beta e_{2}$. For $j=1,2$ set $x_{j}=v\left(e_{j}\right)$ and write $x_{1}=$ $\left(c_{1}, c_{2}, c_{3}\right), x_{2}=\left(d_{1}, d_{2}, d_{3}\right)$. So $\pi_{2}(u)^{2}=|\alpha|^{2}+|\beta|^{2}$, while $\|v\|^{2}=$ $\max _{j=1,2,3}\left|c_{j}\right|^{2}+\left|d_{j}\right|^{2}=1$. The implication Proposition $4.5 \Rightarrow$ Proposition 4.4 follows by noticing that $u$ having norm at most one is equivalent to the inequality (4.1). Similar considerations yield the easier reverse implication.

We do not see a really simple proof of the calculus reformulation of Proposition 4.4 without using Pietsch's factorization theorem. However, a similar reduction of the weaker statement that every 2-dimensional subspace of $\ell_{\infty}^{3}$ has maximal distance to $\ell_{2}^{2}$ produces a calculus statement which is very easy to prove. Indeed, given a 2-dimensional subspace $X$ of $\ell_{\infty}^{3}$, we can choose norm one operators $X \stackrel{u}{\longrightarrow} \ell_{2}^{2}$ and $\ell_{2}^{2} \stackrel{v}{\longrightarrow} X$ so that $d u v=I_{\ell_{2}^{2}}$, where $d$ is the distance from $X$ to $\ell_{2}^{2}$. We can choose the orthonormal basis $e_{1}, e_{2}$ so that 
$1=\|v\|=\left\|v e_{1}\right\|$ and define $x_{1}, x_{2}$, the $c_{i}$ 's, and the $d_{i}$ 's as in the discussion above. Since $\left\|x_{1}\right\|=1$, we can assume, without loss of generality, that $\left|c_{1}\right|=1$. (4.1) holds with $\alpha=\beta=\frac{1}{d}$, and we want to see that this implies that $d \geq \sqrt{2}$; i.e., that $\alpha^{2}+\beta^{2} \leq 1$. So we only need to get $\gamma$ and $\delta$ of modulus one to make the right side of (4.1) one. Since $\left|c_{1}\right|=1, d_{1}=0$, any such choice makes $\left|\gamma c_{1}+\delta d_{1}\right|=1$. Choose $\gamma$ to make $\gamma c_{2} \geq 0$; then $\left|\gamma c_{2}+\delta d_{2}\right| \leq 1$ as long as $\delta d_{2}$ has nonpositive real part, which happens as long as $\delta$ is on a certain closed semicircle. Similarly, $\left|\gamma c_{3}+\delta d_{3}\right| \leq 1$ as long as $\delta$ is on another closed semicircle. Since any two closed semicircles of the unit circle intersect, the desired choice of $\gamma$ and $\delta$ can be made.

Remark 4.6. 1. We can use Propositions 2.6 and 4.4 to prove that $\ell_{\infty}^{3}$ has the 2-summing property. Use the notation of Proposition 2.4 and let $P$ be an orthogonal projection on $L_{2}^{3}(\mu)$. If the rank is one, there is nothing to prove. If the rank is three then we clearly have that $\left\|I_{\infty, 2}\right\|=1$, and if the rank is two, then the proof of Proposition 4.4 implies the result.

2. It is natural to ask if the only subspaces of complex $L_{1}$ with the 2summing property are $\ell_{1}^{2}$ and $\ell_{1}^{3}$. The answer is yes because a subspace of $L_{1}$ of maximal distance is already an $\ell_{1}^{k}$ space. We prove this in the appendix, Proposition 5.7.

\section{APPENDIX}

In this section we present some related results.

Proposition 5.1. Every subspace of complex $\ell_{\infty}^{3}$ is the complexification of a subspace of real $\ell_{\infty}^{3}$

The proof of Proposition 5.1 follows easily from the next two lemmas. Recall that a vector in $\ell_{\infty}^{k}$ is said to be flat if all of its coordinates are unimodular.

Lemma 5.2. Suppose that the subspace $X$ of complex $\ell_{\infty}^{3}$ is not linearly isometric to $\ell_{\infty}^{2}$. Then $X$ contains two linearly independent flat vectors, say $f_{1}$ and $f_{2}$. Moreover, each flat vector in $X$ is of the form $\lambda f_{j}$, where $j \in\{1,2\}$ and $|\lambda|=1$.

Proof. Applying $\ell_{\infty}^{3}$ isometries, we may assume that $X$ is spanned by two vectors of the form $x=(1,0, a)$ and $y=(0,1, b)$ where $a, b \in \mathbb{C}$ with $|a|,|b| \leq 1$. Put $w=x-\psi y$. For $w$ to be flat one needs that $|\psi|=1$ and $|a-\psi b|=1$. Observe that, since $X$ is not linearly isometric to $\ell_{\infty}^{2}$, we have $|a|+|b|>1$; in particular $a b \neq 0$. Thus $\psi \in \mathbb{C}$ should belong to the intersection of the unit circle $\{z:|z|=1\}$ and the circle $\{z:|z-a| b \mid=$ $1 /|b|\}$, hence there are at most two solutions for $\psi$. Thus it will suffice to check that the two circles have a point in common and that they are not tangent at that point. Since the second circle has a bigger radius, this amounts to verifying the strict inequalities

$$
\left|\frac{1}{b}\right|-1<\left|\frac{a}{b}-0\right|<1+\left|\frac{1}{b}\right| \text {. }
$$

These inequalities are obvious, because we have $|a|+|b|>1,|a| \leq 1$ and $|b|>0$.

Lemma 5.3. Suppose that the 2-dimensional subspace $X$ of complex $\ell_{\infty}^{k}$ is spanned by two linearly independent vectors $y, z$ such that $|y|=|z|$. Then 
there is a linear isometry $\Phi$ of $l_{\infty}^{k}$ such that $\Phi y=\overline{\Phi z}$. In particular, $\Phi(X)$ is spanned by two vectors $v, w$, all of whose coordinates are real and which satisfy $\left(|v|^{2}+|w|^{2}\right)^{1 / 2}=|y|$.

Proof. Write $y=\left(y_{1}, y_{2}, \ldots, y_{k}\right)$ and $z=\left(z_{1}, z_{2}, \ldots, z_{k}\right)$. For $j=$ $1,2, \ldots, k$, let $\alpha_{j}$ be a complex number with $\left|\alpha_{j}\right|=1$ such that $\alpha_{j} y_{j}=\overline{\alpha_{j} z_{j}}$. Such numbers obviously exist, we may also impose the condition $\Re \alpha_{j} y_{j} \geq 0$. Now the isometry $\Phi$ can be defined by the formula $\Phi\left(x_{1}, x_{2}, \ldots, x_{k}\right)=$ $\left(\alpha_{1} x_{1}, \alpha_{2} x_{2}, \ldots, \alpha_{k} x_{k}\right)$. Clearly, the vectors $v=\frac{1}{2}(\Phi y+\Phi z)$ and $w=$ $\frac{1}{2 i}(\Phi y-\Phi z)$ have the required property.

Propositions 2.9, 2.10, and 5.1 suggest an alternate method for proving Proposition 4.4 since they combine to take care of the case where the operator achieves its norm at two "flat" vectors:

Lemma 5.4. Let $X$ be a 2-dimensional subspace of complex $\ell_{\infty}^{3}$ and $T$ a complex-linear operator from $X$ into a Hilbert space such that $\|T x\|=\|T y\|$, where $x, y$ are linearly independent vectors in $X$ for which $|x|=|y|$. Then $\pi_{2}(T)=\|T\|$.

Proof. In view of Lemma 5.3 we can assume that there are vectors $v, w$ in $X$ all of whose coordinates are real for which $|v|^{2}+|w|^{2}=|x|^{2}, x=v+i w$, and $y=v-i w$. Thus if we let $E$ be the collection of real-linear combinations of $\{v, w\}$, we can regard $X$ as the complexification $E_{\mathbb{C}}$ of $E$. The assumption on $\{x, y\}$ means that the pair $\{v, w\}$ satisfies condition (iv) in Proposition 2.11, hence condition (i) of Proposition 2.11 says that $T$ is the complexification of the restriction of $T$ to $E$, whence by Proposition $2.10, \pi_{2}(T)=\|T\|$.

The next lemma takes care of the case where the operator achieves its norm at a non-flat vector.

Lemma 5.5. Suppose that $X$ is a 2-dimensional subspace of complex $\ell_{\infty}^{3}$ and the norm one operator $X \stackrel{T}{\longrightarrow} \ell_{2}^{2}$ achieves its norm at a non-flat vector $x=$ $\left(x_{1}, x_{2}, x_{3}\right)$ on the unit sphere of $X$. Then there are norm one operators $X \stackrel{V}{\longrightarrow} \ell_{\infty}^{2}$ and $\ell_{\infty}^{2} \stackrel{W}{\longrightarrow} \ell_{2}^{2}$ so that $T=W V$. Consequently, by Example 2.2, $\pi_{2}(T)=1$.

Proof. Since the result is trivial if $T$ has rank one, we assume that $T$ has rank two. This implies that two coordinates of $x$, say, $x_{1}$ and $x_{2}$, are unimodular. [Indeed, suppose, for example, that $\left|x_{2}\right|$ and $\left|x_{3}\right|$ are both less than $1-\epsilon$. Take $y$ in $X$ with $y_{1}=0$ and $0<\|y\|<\epsilon$, so $\|x \pm y\|=1$. But since $\ell_{2}$ is strictly convex, $\|T(x+\eta y)\|>\|T x\|$ for either $\eta=1$ or $\eta=-1$.] We may also assume that $X$ contains two vectors, say $y, w$, such that $y=\left(1,0, y_{3}\right)$ and $w=\left(0,1, w_{3}\right)$. (Otherwise, $X$ is spanned by two vectors with disjoint supports and the conclusion of the lemma is obvious.) Let $\Psi$ be the function defined for $z \in \mathbb{C}$ by the formula

$$
\Psi(z)=\left\|T\left(x_{1} y+z w\right)\right\|^{2} .
$$

Observe that $\Psi(z)=\left\|T\left(x_{1} y\right)+z T w\right\|^{2}$ is a quadratic function of $(\Re z, \Im z)$, which at infinity is asymptotically equal to $m|z|^{2}$, where $m=\|T w\|^{2}>0$. It follows that there is a number $z_{0} \in \mathbb{C}$ such that $\Psi(z)=m\left|z-z_{0}\right|^{2}+\Psi\left(z_{0}\right)$ for every $z \in \mathbb{C}$. It is obvious now that either $z_{0}=0$, so that $\Psi$ is constant on 
the unit circle, or else $\Psi$ has a unique local maximum on the unit circle (which must also be the global maximum of $\Psi$ on the circle). Note that at $z=x_{2}$ the function $\Psi$ does have a local maximum. In each case it follows that, for every $z$ with $|z| \leq 1$, we have $\Psi(z) \leq \Psi\left(x_{2}\right)=1$.

Put $V\left(z_{1} y+z_{2} w\right)=\left(z_{1}, z_{2}\right)$. Then $V: X \rightarrow l_{\infty}^{2}$ and $\|V\| \leq 1$. The latter property of $\Psi$ can be restated as follows: if $\left(z_{1}, z_{2}\right) \in \mathbb{C}^{2},\left|z_{1}\right|=\left|z_{2}\right|=1$, and $u=z_{1} / x_{1}$, then

$$
\left\|T\left(z_{1} y+z_{2} w\right)\right\|=\sqrt{\Psi\left(z_{2} / u\right)} \leq 1=\max \left\{\left|z_{1}\right|,\left|z_{2}\right|\right\}=\left\|V\left(z_{1} y+z_{2} w\right)\right\| .
$$

Since $W=T V^{-1}$ attains its norm at an extreme point, we have just checked that $\|W\| \leq 1$.

Now we can give an alternate:

Proof of Proposition 4.4. Suppose that the 2-dimensional subspace $X$ of complex $\ell_{\infty}^{3}$ fails the 2-summing property. Let $T_{0}$ be a norm one linear mapping of $X$ into a Hilbert space $H$ whose 2-summing norm is maximal among all norm one linear maps of $X$ into $H$. Thus $\pi_{2}\left(T_{0}\right)>\left\|T_{0}\right\|=1$; in particular, $T_{0}$ is of rank $>1$.

By Lemma 5.5, $T_{0}$ does not attain its norm at any non-flat vector. Hence, if $f_{1}, f_{2} \in X$ are the two flat vectors described in Lemma 5.2, then $\left\|T_{0}\right\|=$ $\max \left\{\left\|T_{0} f_{1}\right\|,\left\|T_{0} f_{2}\right\|\right\}$. Using Lemma 5.4, we rule out the possibility that $\left\|T_{0} f_{1}\right\|=\left\|T_{0} f_{2}\right\|$.

Assume that $\left\|T_{0}\right\|=\left\|T_{0} f_{1}\right\|>\left\|T_{0} f_{2}\right\|$. Observe that for every $\epsilon>0$ there is an operator $T_{\epsilon}: X \rightarrow H$ such that

$$
\left\|T_{\epsilon}-T_{0}\right\|<\epsilon, \quad\left\|T_{\epsilon} f_{1}\right\|=\left\|T_{0} f_{1}\right\|
$$

and the inequality $\left\|T_{\epsilon} x\right\| \leq\left\|T_{0} x\right\|$ is possible only if $x=\lambda f_{1}$.

Since rank $T$ is $>1$, the latter property of $T_{\epsilon}$ implies that $\pi_{2}\left(T_{\epsilon}\right)>\pi_{2}\left(T_{0}\right)$. By the maximality of $\pi_{2}\left(T_{0}\right)$, we infer that $\left\|T_{\epsilon}\right\|>1$. However, $T_{\epsilon}$ does attain its norm somewhere and it cannot happen at any flat vector, because as soon as $\epsilon<\left\|T_{0} f_{1}\right\|-\left\|T_{0} f_{2}\right\|$ we have $\left\|T_{\epsilon} f_{2}\right\|<\left\|T_{\epsilon} f_{1}\right\|=1$. Using Lemma 5.5 again, we infer that $\pi_{2}\left(T_{\epsilon}\right)=\left\|T_{\epsilon}\right\|$. Now, letting $\epsilon$ tend to 0 , we obtain that $\pi_{2}\left(T_{0}\right)=\left\|T_{0}\right\|$, which contradicts our initial assumption.

To find 3-dimensional subspaces other than $\ell_{\infty}^{3}$ and $\ell_{1}^{3}$ which have the 2summing property, it is natural to look inside $\ell_{\infty}^{4}$. However:

Proposition 5.6. Let $X$ be a 3-dimensional subspace of complex $\ell_{\infty}^{4}$ not isometric to $\ell_{\infty}^{3}$. Then $X$ does not have the 2-dimensional 2-summing property.

Proof. First notice that without loss of generality $X$ is spanned by three vectors of the form $\left(1,0,0, a_{1}\right),\left(0,1,0, a_{2}\right),\left(0,0,1, a_{3}\right)$ with $a_{1}, a_{2}, a_{3}$ nonnegative real numbers satisfying $a_{1}, a_{2}, a_{3} \leq 1$ and $a_{1}+a_{2}+a_{3}>1$. Indeed, let $\left(b_{1}, b_{2}, b_{3}, b_{4}\right)$ be a non-zero vector annihilating $X$ and assume $\left|b_{4}\right| \geq\left|b_{1}\right|,\left|b_{2}\right|,\left|b_{3}\right|$. Applying $\ell_{\infty}^{4}$ isometries we may assume that $b_{i} / b_{4}$ are non-positive reals. Put $a_{i}=-b_{i} / b_{4}$. Note that, since $X$ is not isometric to $\ell_{\infty}^{3}, a_{1}+a_{2}+a_{3}>1$. 
Fix $\alpha, \beta, \gamma, \delta$ non-negative real numbers and $\varphi, \psi \in \mathbb{C}$ with $|\varphi|=|\psi|=1$ and consider the following two vectors in $X$ :

$$
\begin{aligned}
& x=\left(\alpha, \varphi, \gamma \psi, \alpha a_{1}+\varphi a_{2}+\gamma \psi a_{3}\right), \\
& y=\left(\beta, 0,-\delta \psi, \beta a_{1}-\delta \psi a_{3}\right) .
\end{aligned}
$$

We are going to show that, for some choice of the parameters, $|x|^{2}+|y|^{2}$ is constantly equal to one while for the same choice span $\{x, y\}$ does not contain a flat vector. Once this is proved one concludes the proof as in Example 2.3.

$|x|^{2}+|y|^{2} \equiv 1$ is equivalent to

$$
1=\alpha^{2}+\beta^{2}=\gamma^{2}+\delta^{2}=\left|\alpha a_{1}+\varphi a_{2}+\gamma \psi a_{3}\right|^{2}+\left|\beta a_{1}-\delta \psi a_{3}\right|^{2}
$$

while, if $|a x+b y| \equiv 1$, we may assume without loss of generality that $a=1$ and then

$$
1=|\alpha+b \beta|=|\gamma-b \delta|=\left|\alpha a_{1}+\varphi a_{2}+\gamma \psi a_{3}+b\left(\beta a_{1}-\delta \psi a_{3}\right)\right| .
$$

Note that if $\beta \neq 0$ then the first equations in (5.1) and (5.2) imply that $2 \frac{\alpha}{\beta} \Re b=$ $1-|b|^{2}$. Similarly, if $\delta \neq 0,-2 \frac{\gamma}{\delta} \Re b=1-|b|^{2}$. It follows that $|b|=1$. If in addition to $\beta, \delta>0$ also $\alpha>0$ or $\gamma>0$ then $b= \pm i$ and the last equations in (5.1) and (5.2) imply that $\alpha a_{1}+\varphi a_{2}+\gamma \psi a_{3}$ and $\beta a_{1}-\delta \psi a_{3}$ are pointing in the same or opposite directions. Thus, it is enough to find non-negative reals $\alpha, \beta, \gamma, \delta$ with all but possibly $\alpha$ or $\gamma$ positive and complex numbers $\varphi, \psi$ of modulus one satisfying (5.1) but such that $\alpha a_{1}+\varphi a_{2}+\gamma \psi a_{3}$ and $\beta a_{1}-\delta \psi a_{3}$ are not pointing in the same or opposite directions.

Assume first that $a_{2}^{2}+\left(a_{1}-a_{3}\right)^{2}<1$ and $a_{2}>0$. Clearly there are $0<\alpha, \gamma<1$ and $0<\varphi<\pi$ for which $\left|\alpha a_{1}+\varphi a_{2}+\gamma a_{3}\right|>1$. Then also $\left|\alpha a_{1}+\varphi a_{2}+\gamma a_{3}\right|^{2}+\left|\left(1-\alpha^{2}\right)^{1 / 2} a_{1}-\left(1-\gamma^{2}\right)^{1 / 2} a_{3}\right|^{2}>1$. Replacing $\alpha, \gamma$ with $t \alpha, t \gamma$ for some $0<t<1$ we find $0<\alpha, \gamma<1$ and $0<\varphi<\pi$ for which

$$
\left|\alpha a_{1}+\varphi a_{2}+\gamma a_{3}\right|^{2}+\left|\left(1-\alpha^{2}\right)^{1 / 2} a_{1}-\left(1-\gamma^{2}\right)^{1 / 2} a_{3}\right|^{2}=1 .
$$

Clearly, $\alpha a_{1}+\varphi a_{2}+\gamma a_{3}$ and $\left(1-\alpha^{2}\right)^{1 / 2} a_{1}-\left(1-\gamma^{2}\right)^{1 / 2} a_{3}$ are not pointing in the same or opposite direction.

If $a_{\pi(2)}^{2}+\left(a_{\pi(1)}-a_{\pi(3)}\right)^{2} \geq 1$ for all permutations, $\pi$, of the indices $1,2,3$ for which $a_{\pi(2)}>0$ then, assuming as we may that $a_{1} \geq a_{2}, a_{3}$ and $a_{1}>0$, it is easily checked that there are $\alpha, \beta>0$ with $\alpha^{2}+\beta^{2}=1$ for which

$$
\left|\alpha a_{1}+a_{2}\right|^{2}+\left|\beta a_{1}+a_{3}\right|^{2}>1>\left|\alpha a_{1}-a_{2}\right|^{2}+\left|\beta a_{1}-a_{3}\right|^{2} \text {. }
$$

Indeed,

$$
1 \leq\left(a_{1}^{2}+\left(a_{2}-a_{3}\right)^{2}\right)^{1 / 2}<a_{1}+\left(a_{2}^{2}+a_{3}^{2}\right)^{1 / 2}=\sup _{\alpha^{2}+\beta^{2}=1}\left(\left|\alpha a_{1}+a_{2}\right|^{2}+\left|\beta a_{1}+a_{3}\right|^{2}\right)^{1 / 2} .
$$

Moreover, the sup is attained for $(\alpha, \beta)$ proportional to $\left(a_{2}, a_{3}\right)$. For this choice of $(\alpha, \beta),\left|\alpha a_{1}-a_{2}\right|^{2}+\left|\beta a_{1}-a_{3}\right|^{2}=\left(a_{1}-\left(a_{2}^{2}+a_{3}^{2}\right)^{1 / 2}\right)^{2}<1$. Choose now $\gamma=0, \delta=1$ and notice that there is a one parameter family of $\varphi, \psi$ for which $\left|\alpha a_{1}+\varphi a_{2}\right|^{2}+\left|\beta a_{1}-\psi a_{3}\right|^{2}=1$ but not for all of members of this family do $\alpha a_{1}+\varphi a_{2}$ and $\beta a_{1}-\psi a_{3}$ point in the same or opposite directions.

Remark. It is easy to adjust the proof above to show that for $n>3$ no $n$ dimensional subspace of $\ell_{\infty}^{n+1}$ has the 2-summing property. Indeed without 
loss of generality any such subspace is spanned by $n$ vectors of the form $\left(1,0, \ldots, 0, a_{1}\right), \ldots,\left(0, \ldots, 0,1, a_{n}\right)$ with $0 \leq a_{i} \leq 1$. If $\sum a_{i} \leq 1$ the subspace is isometric to $\ell_{\infty}^{n}$ which does not have the 2 -summing property. Otherwise these $n$ vectors can be blocked to get three vectors which could replace the three vectors with which we started the proof above.

We next present a proof that all maximal distance subspaces of $L_{1}$ are $\ell_{1}^{n}$ spaces. In particular there are no new subspaces of (real or complex) $L_{1}$ with the 2-summing property. Essentially the same proof shows that all maximal distance subspaces of $L_{p}$ are $\ell_{p}^{n}$ spaces, $1 \leq p<\infty$. This fact is not new: it was observed by J. Bourgain that the case $p=1$ follows from [FJ]. The case $1<p<2$ was first proved in [BT]. Komorowski [Ko] was the first to prove the $2<p<\infty$ case. The proof here is very similar to Komorowski's but includes also the $1 \leq p<2$ case.

Proposition 5.7. Let $1 \leq p<\infty$ and let $X$ be $n$-dimensional subspace of an $L_{p}(\mu)$ space with $d\left(X, \ell_{2}^{n}\right)=n^{\left|\frac{1}{p}-\frac{1}{2}\right|}$. Then $X$ is isometric to $\ell_{p}^{n}$.

Proof. By Lewis' theorem [L] we may assume that $\mu$ is a probability measure and that $X$ has a basis $x_{1}, x_{2}, \ldots, x_{n}$ satisfying

$$
\sum_{i=1}^{n}\left|x_{i}\right|^{2} \equiv 1
$$

and

$$
\int\left|\sum_{i=1}^{n} a_{i} x_{i}\right|^{2} d \mu=\frac{1}{n} \sum_{i=1}^{n}\left|a_{i}\right|^{2} \quad \text { for all scalars. }
$$

Then, for $1 \leq p \leq 2$,

$$
\frac{1}{n^{1 / 2}}\left(\sum_{i=1}^{n}\left|a_{i}\right|^{2}\right)^{1 / 2}=\left(\int\left|\sum_{i=1}^{n} a_{i} x_{i}\right|^{2} d \mu\right)^{1 / 2} \geq\left(\int\left|\sum_{i=1}^{n} a_{i} x_{i}\right|^{p} d \mu\right)^{1 / p}
$$

and

$$
\begin{aligned}
\frac{1}{n^{1 / 2}}\left(\sum_{i=1}^{n}\left|a_{i}\right|^{2}\right)^{1 / 2} & =\left(\int\left|\sum_{i=1}^{n} a_{i} x_{i}\right|^{2} d \mu\right)^{1 / 2} \\
& \leq\left(\int\left|\sum_{i=1}^{n} a_{i} x_{i}\right|^{p} d \mu\right)^{1 / 2} \sup \left|\sum_{i=1}^{n} a_{i} x_{i}\right|^{\frac{2-p}{2}} \\
& \leq\left(\int\left|\sum_{i=1}^{n} a_{i} x_{i}\right|^{p} d \mu\right)^{1 / 2}\left(\sum_{i=1}^{n}\left|a_{i}\right|^{2}\right)^{\frac{2-p}{4}}
\end{aligned}
$$

(by (5.3)). Thus

$$
\frac{1}{n^{1 / 2}}\left(\sum_{i=1}^{n}\left|a_{i}\right|^{2}\right)^{p / 4} \leq\left(\int\left|\sum_{i=1}^{n} a_{i} x_{i}\right|^{p} d \mu\right)^{1 / 2}
$$

and

$$
\frac{1}{n^{1 / p}}\left(\sum_{i=1}^{n}\left|a_{i}\right|^{2}\right)^{1 / 2} \leq\left(\int\left|\sum_{i=1}^{n} a_{i} x_{i}\right|^{p} d \mu\right)^{1 / p}
$$


This shows that, if $T$ is the map sending the $x_{i}$ 's to an orthonormal basis, then $\|T\|\left\|T^{-1}\right\| \leq n^{\left|\frac{1}{p}-\frac{1}{2}\right|}$. It follows that there are $a_{1}, a_{2}, \ldots, a_{n}$ for which equality is achieved in both (5.5) and (5.6).

If $2<p<\infty$, then we get similarly that

$$
\frac{1}{n^{1 / 2}}\left(\sum_{i=1}^{n}\left|a_{i}\right|^{2}\right)^{1 / 2} \leq\left(\int\left|\sum_{i=1}^{n} a_{i} x_{i}\right|^{p} d \mu\right)^{1 / p}
$$

and

$$
\begin{aligned}
\left(\int\left|\sum_{i=1}^{n} a_{i} x_{i}\right|^{p} d \mu\right)^{1 / p} & \leq\left(\int\left|\sum_{i=1}^{n} a_{i} x_{i}\right|^{2} d \mu\right)^{1 / p} \sup \left|\sum_{i=1}^{n} a_{i} x_{i}\right|^{\frac{p-2}{p}} \\
& \leq\left(\int\left|\sum_{i=1}^{n} a_{i} x_{i}\right|^{2} d \mu\right)^{1 / p}\left(\sum_{i=1}^{n}\left|a_{i}\right|^{2}\right)^{\frac{p-2}{2 p}} \\
& =\frac{1}{n^{1 / p}}\left(\sum_{i=1}^{n}\left|a_{i}\right|^{2}\right)^{1 / 2} .
\end{aligned}
$$

Again we get that some $a_{1}, a_{2}, \ldots, a_{n}$ must satisfy $\left(5.5^{\prime}\right)$ and $\left(5.6^{\prime}\right)$ as equalities. Examining when equalities can occur in $(5.5),\left(5.5^{\prime}\right),(5.6)$ and $\left(5.6^{\prime}\right)$, we see that for all $p$ there are $a_{1}, a_{2}, \ldots, a_{n}$ such that $\left|\sum_{i=1}^{n} a_{i} x_{i}\right|$ is a constant on its support, $A$, and the constant must be $\left(\sum_{i=1}^{n}\left|a_{i}\right|^{2}\right)^{1 / 2}$ which we may assume is equal to 1 . Moreover, on $A,\left(x_{1}(t), x_{2}(t), \ldots, x_{n}(t)\right)$ must be equal to $\theta(t)\left(\bar{a}_{1}, \bar{a}_{2}, \ldots, \bar{a}_{n}\right)$ for some function $\theta$ satisfying $|\theta(t)| \equiv 1$. Applying a space isometry, we may assume that $\theta \equiv 1$ and then $\sum_{i=1}^{n} a_{i} x_{i}=\chi_{A}$. Note also that $\mu(A)=\int\left|\sum_{i=1}^{n} a_{i} x_{i}\right|^{2} d \mu=\frac{1}{n}$. We thus get that we may assume that $\chi_{A} \in X$. Since each $x_{i}$ is constant on $A$, we get that for all $f \in X, f_{\mid A}$ is a constant and $f_{\mid A^{c}}$ also belongs to $X$. Put

$$
Y=\left\{f_{\mid A^{c}}: \quad f \in X\right\}
$$

then $Y \subset X$ and $\operatorname{dim} Y=n-1$. Necessarily $d\left(Y, \ell_{p}^{n-1}\right)=(n-1)^{\left|\frac{1}{p}-\frac{1}{2}\right|}$ and continue.... .

\section{REFERENCES}

[B] D. P. Blecher, Tensor products of operator spaces II, Canad. J. Math. 44 (1992), 75-90.

[BM] B. Bagchi and G. Misra, Contractive homomorphisms and tensor product norms, preprint.

[BT] J. Bourgain and L. Tzafriri, Invertibility of "large" submatrices with applications to the geometry of Banach spaces and harmonic analysis, Israel J. Math. 57 (1987), 137-224.

[Dv] A. Dvoretzky, Some results on convex bodies and Banach spaces, Proc. Sympos. Linear Spaces, Jerusalem, 1961, pp. 123-160.

[FJ] T. Figiel and W. B. Johnson, Large subspaces of $l_{\infty}^{n}$ and estimates of the Gordon-Lewis constant, Israel J. Math. 37 (1980), 92-112.

[J] F. John, Extremum problems with inequalities as subsidiary conditions, Courant Anniversary Volume, Interscience, New York, 1948, pp. 346-361.

[K] S. V. Kisliakov, On spaces with "small" annihilators, Zap. Nauchn. Sem. Leningrad Otdel. Math. Inst. Steklov (LOMI) 65 (1976), 192-195.

[Ko] R. Komorowski, Isometric characterizations of $\ell_{p}^{n}$ spaces, Canad. J. Math. (to appear).

[L] D. R. Lewis, Ellipsoids defined by Banach ideal norms, Mathematika 26 (1979), 18-29. 
[LT1] J. Lindenstrauss and L. Tzafriri, Classical Banach spaces I, Sequence spaces, Springer-Verlag, Berlin, 1977.

[LT2] _ Classical Banach spaces II, Function spaces, Springer-Verlag, Berlin, 1979.

[MW] V.D. Milman and H. Wolfson, Spaces with extremal distances from the Euclidean space, Israel J. Math. 29 (1978), 113-131.

[Pa] V. I. Paulsen, Representations of function algebras, abstract operator spaces, and Banach space geometry, J. Funct. Anal. 109 (1992), 113-129.

[Pi] G. Pisier, Une nouvelle classe d'espaces vérifiant le théorème de Grothendieck, Ann. Inst. Fourier (Grenoble) 28 (1978), 69-90.

[T-J1] N. Tomczak-Jaegermann, Computing 2-summing norms with few vectors, Ark. Mat. 17 (1979), 273-277.

[T-J2] __ Banach-Mazur distances and finite-dimensional operator ideals, Pitman Monographs and Surveys in Pure and Applied Mathematics, 38, Longman, 1989.

Division of Mathematics, Computer Science and Statistics, The University of Texas at San Antonio, San Antonio, Texas 78249

E-mail address: arias@ringer.cs.utsa.edu

Institute of Mathematics, Polish Academy of Sciences, Gdansk, Poland

E-mail address: tfigiel@impan.impan.gov.pl

Department of Mathematics, Texas A\&M University, College Station, Texas 77843

E-mail address: johnson@math.tamu.edu

Department of Theoretical Mathematics, The Weizmann Institute of Science, ReHOVOT, ISRAEL

E-mail address: mtschech@weizmann.weizmann.ac.il 